\title{
QUANTUM GROUP STRUCTURE AND LOCAL FIELDS IN THE ALGEBRAIC APPROACH TO 2D GRAVITY
}

\author{
Jens SCHNITTGER \\ CERN-TH, 1211 Geneva 23, Switzerland.
}

\begin{abstract}
This review contains a summary of work by J.-L. Gervais and the author on the operator approach to $2 \mathrm{~d}$ gravity. Special emphasis is placed on the construction of local observables -the Liouville exponentials and the Liouville field itself - and the underlying algebra of chiral vertex operators. The double quantum group structure arising from the presence of two screening charges is discussed and the generalized algebra and field operators are derived. In the last part, we show that our construction gives rise to a natural definition of a quantum tau function, which is a noncommutative version of the classical group-theoretic representation of the Liouville fields by Leznov and Saveliev.
\end{abstract}




\section{Introduction}

Two-dimensional gravity has been the subject of intensive study in recent years. The approaches which were employed cover a wide spectrum, ranging from topological theory to matrix models, and this lead to the discovery of fruitful relations and cross-connections. Each of the methods has its own particular merits and shortcomings, and so the preference of one over the other depends to some extent on the nature of the question we want to address. The traditional virtue of the operator approach is to provide insight into the local structure of the theory, i.e. into operator algebra and correlation functions, instead of fusion rules and amplitudes only. This may not be so important if we are only interested in the calculation of noncritical string scattering amplitudes, but is evidently crucial if we want to understand the structure of $2 \mathrm{~d}$ gravity/Liouville theory as a conformal field theory. The most spectacular success produced so far by the algebraic method was the discovery of the underlying quantum group symmetry [1] [2][6], which had remained completely invisible in the other approaches. Until recent times, the main technical tool in this framework was the detailed study of the Virasoro null vector equations and their monodromy properties [1]-9], which in turn are related to representations of the $U_{q}(s l(2))$ quantum group with positive half-integer spins [8] 9]. More precisely, one finds that a subset of the observables of the theory - the positive integer powers of the inverse square root of the metric - are described by chiral vertex operators which are degenerate fields in the sense of BPZ13. However, the study of representations with positive half-integer spins does not by far answer all the physical questions we want to ask about $2 \mathrm{D}$ gravity. For instance, the coupling of minimal matter to gravity requires negative half-integer spins, and modular invariance in the strong coupling version of the theory forces us to consider operators with quantum-group spins which are rational, but not halves of integers 17]. Of course the full operator content of the theory, and in particular the possibility of defining the Liouville field itself, is explored only if we can define the Liouville exponential with continuous $J$, so that we may let $\Phi=-\left.\frac{d}{d J}\right|_{J=0} \exp \left(-J \alpha_{-} \Phi\right) / \alpha_{-}$. The basic difficulty in going away from positive half-integer spins is that one no longer deals with degenerate fields satisfying null-vector equations. We will show how to solve this problem by introducing an operator Coulomb-gas realization of the chiral vertex operators. This allows us to establish their exchange algebra, which is the essential ingredient for the reconstruction of local observables. The crucial observation in this context is that the braiding problem, due to its essentially topological nature, can be represented in terms of simple quantum-mechanical variables.

Another interesting aspect of Liouville theory is its connection with the general theory of integrable systems. As the simplest in the sequence of $A_{n}$ Toda theories, it should possess both a description and a multi-time generalization in terms of Toda lattice $\tau$ functions and their reductions. Though this is completely understood classically, even the very concept of a $\tau$ function is still under discussion on the quantum level [36] 37] [39] [40] 41]. As a contribution to this discussion, we present an argument why our quantum exponentials should possess an interpretation as quantum $\tau$ functions. The analysis is based on the (classical) group-theoretical 
representation of the Liouville exponentials by Leznov and Saveliev[34], which is known to have the properties of a $\tau$ function. Due to the explicit control furnished by the operator approach over the quantization of all the defining elements of this representation, we are able to write the natural quantum equivalent of the LeznovSaveliev $\tau$ function.

This review is organized as follows. In section 2 we recall some elementary background material. Section 3 is devoted to the case of a single screening charge, where the quantum group structure is known to be the standard $U_{q}(s l(2))$. We work with Coulomb-gas type operators which have a diagonal monodromy, i.e. well-defined periodicity properties, instead of explicitly quantum group covariant operators. This picture has the advantage that the chiral exchange algebra can be derived using only free field methods, as we will show. The fusion algebra (operator product) is not as easily accessible by this method, but can be obtained from the braiding algebra if one assumes the validity of the general Moore-Seiberg 15 proportionality relation between fusing and braiding matrices. The control over the chiral algebra then allows us to construct Liouville exponentials for arbitrary $J$, and we give an expression for the Liouville field itself. The validity of the canonical equal-time commutation relations as well as the quantum equations of motion is demonstrated. We discuss the periodicity properties of our definition of the Liouville exponentials resp. of the Liouville field and their connection with the presence of singularities in the elliptic sector. The preservation on the quantum level of the symmetry under the exchange of the two equivalent Bäcklund free fields 19 -which has the meaning of a Weyl reflection- is derived for half-integer spins. In section 4, we consider both screening charges together. The corresponding quantum group structure $U_{q}(\operatorname{sl}(2)) \odot U_{\widehat{q}}(\operatorname{sl}(2))$ is known 8 to be given by a sort of graded tensor product in the degenerate case, where the primary fields of spins $J$ and $\widehat{J}$ are of the type $(2 \widehat{J}+1,2 J+1)$ in the BPZ classification. This is because the fields of type $(1,2 J+1)$ field commute with those of type $(2 \widehat{J}+1,1)$ up to a simple phase. For continous spins, however, the situation is different, and the notion of two independent spins $J$ and $\widehat{J}$ becomes meaningless. The latter two unite into a single parameter $J^{e}:=J+\widehat{J} \pi / h(h$ is defined by $q=\exp (i h)$ as usual), and a new factorization property of the fusion and braiding matrices in terms of the two screening charges emerges. The new $U_{q \widehat{q}}(s l(2))$ structure can be described in terms of "vectorial" $6 \mathrm{j}$ symbols which have been shown in [17] to satisfy all of the polynomial equations of Moore and Seiberg 15]. The corresponding representations have one spin but two magnetic quantum numbers, corresponding to the two types of screening charges.

In the last section, we consider the integrability structure of the theory from the $\tau$ function point of view. We start from the group-theoretical representation of the classical Liouville exponentials by Leznov and Saveliev [34], which can be understood as a classical tau function and in particular fulfills bilinear equations of the Hirota type. The quantum group covariant quantization scheme described here provides a natural prescription for the proper quantization of the Leznov-Saveliev formula, such that its algebraic and group theoretical properties are just the obvious q-deformations of those of the classical case. For this purpose it is most natural to work with an explicitly quantum group covariant operator basis [2] [6] [9], where fusion 
and braiding are given in terms of $3 j$ symbols and universal $R$-matrix.

\section{Some background material}

Let us rederive some background material about Liouville theory, in order to introduce the coming discussion. The solutions of the classical Liouville dynamics, which is described by the action

$$
S=\frac{1}{8 \pi} \int d \tau d \sigma\left\{\left(\partial_{\tau} \Phi\right)^{2}-\left(\partial_{\sigma} \Phi\right)^{2}-\mu^{2} e^{2 \sqrt{\gamma} \Phi}\right\}
$$

are given by

$$
2 \sqrt{\gamma} \Phi=\ln \left[\frac{8}{\mu^{2} \sqrt{\gamma}} \frac{A^{\prime}(u) B^{\prime}(v)}{(A(u)-B(v))^{2}}\right] \quad, \quad u=\tau+\sigma, \quad v=\tau-\sigma
$$

with $A$ and $B$ arbitrary functions, and $\sigma \in[0,2 \pi]$. The coupling constant is noted $\gamma$. We have redefined $\Phi \rightarrow 2 \sqrt{\gamma} \Phi$ in order to agree with the classical limit of standard quantum normalizations, where $2 \sqrt{\gamma}$ is the limit of the screening charge $\alpha_{-}$. Eq.2.2 is invariant under the projective transformations

$$
A \rightarrow \frac{a A+b}{c A+d} \quad B \rightarrow \frac{a B+b}{c B+d}
$$

$a, b, c, d$ complex, which on the quantum level gives rise to the $U_{q}(s l(2))$ quantum group symmetry. Introducing the chiral fields

$$
\begin{aligned}
& f_{m}^{(J)}=\sqrt{\left(\begin{array}{c}
2 J \\
J+m
\end{array}\right)}\left(A^{\prime-1 / 2}\right)^{J-m}\left(A A^{\prime-1 / 2}\right)^{J+m}, \\
& \bar{f}_{m}^{(J)}=\sqrt{\left(\begin{array}{c}
2 J \\
J+m
\end{array}\right)}\left(B^{\prime-1 / 2}\right)^{J+m}\left(B B^{\prime-1 / 2}\right)^{J-m},
\end{aligned}
$$

we can write the Liouville exponentials as

$$
e^{-2 J \sqrt{\gamma} \Phi}=\left(\frac{\mu^{2} \sqrt{\gamma}}{8}\right)^{J} \sum_{m=-J}^{J}(-1)^{J+m} f_{m}^{(J)} \bar{f}_{m}^{(J)}
$$

for any positive half-integer $J$. It is easy to verify that, when $A$ and $B$ undergo the Möbius transformation Eq.2.3, the functions $f_{m}^{(J)}, \bar{f}_{m}^{(J)}$ transform as standard (finitedimensional) spin $J$ representations of $\operatorname{sl}(2, \mathbf{C})$, and Eq.2.5 is the corresponding singlet. For general $J$, Eq.2.5 is still valid formally, with the $m$-sum extending to $+\infty$ or to $-\infty$, depending on whether we work with the semi-infinite representations with $J+m$ positive integer, or $J-m$ positive integer. In practice, Eq.2.5 is an expansion in $(A / B)^{m}$, and highest-weight representations, with $-\infty \leq m \leq J$ (resp. lowest-weight representations, with $-J \leq m \leq \infty)$ will give a convergent

\footnotetext{
${ }^{1}$ In this formula, contrary to the rest of the article, we of course use ordinary binomial coefficients.
} 
expansion for $|A|>|B|$ (resp. $|B|>|A|$ ). Both choices should represent the same function, since they are just related by the particular $s l(2)$-transformation

$$
A \rightarrow-1 / A \quad B \rightarrow-1 / B \quad
$$

which sends $m \rightarrow-m$ in $f_{m}^{(J)}, \bar{f}_{m}^{(J)}$. $]$ Of course, in the case of positive half-integer $J$, this amounts only to a trivial permutation of terms in the sum Eq.2.5. For continous $J$, however, the highest resp. lowest weight representations are representations only of the algebra but not of the group, due to the multivaluedness of the $f_{m}^{(J)}$ under the group operations Eq.2.3. Consequently, the transformation Eq.2.6 exchanges highest and lowest weight representations. From the general point of view of Toda theory, it can be regarded as representing the Weyl group symmetry[18].

Periodicity of $\Phi$ implies that $A$ and $B$ must be periodic up to a projective transformation, which is called the monodromy matrix. In the elliptic and hyperbolic sectors of the theory, we can always pick a representative of the equivalence class defined by Eq.2.3 such that the monodromy matrix is diagonal, i.e. such that $A$ and $B$ are periodic up to a multiplicative constant. In fact, there are precisely two such representatives, related by Eq.2.6 which can thus be viewed in this context as a kind of residual symmetry. It is then possible to define two equivalent sets of chiral free fields by

$$
\begin{array}{rr}
\sqrt{\gamma} \vartheta_{1}(u):=\ln A^{\prime-1 / 2}(u), & \sqrt{\gamma} \bar{\vartheta}_{1}(v):=\ln B B^{\prime-1 / 2}(v), \\
\sqrt{\gamma} \vartheta_{2}(u):=\ln A A^{\prime-1 / 2}(u), & \sqrt{\gamma} \bar{\vartheta}_{2}(v):=\ln B^{\prime-1 / 2}(v)
\end{array}
$$

Indeed, one may show that the canonical Poisson brackets of Liouville theory give the following free-field Poisson bracket relations

$$
\left\{\vartheta_{1}^{\prime}\left(\sigma_{1}\right), \vartheta_{1}^{\prime}\left(\sigma_{2}\right)\right\}_{\text {P.B. }}=\left\{\vartheta_{2}^{\prime}\left(\sigma_{1}\right), \vartheta_{2}^{\prime}\left(\sigma_{2}\right)\right\}_{\text {P.B. }}=2 \pi \delta^{\prime}\left(\sigma_{1}-\sigma_{2}\right)
$$

with similar relations for the bar components. The explicit mode expansions in terms of zero modes and oscillators are given by

$$
\begin{aligned}
& \vartheta_{j}(u)=q_{0}^{(j)}+p_{0}^{(j)} u+i \sum_{n \neq 0} e^{-i n u} p_{n}^{(j)} / n, \\
& \bar{\vartheta}_{j}(v)=\bar{q}_{0}^{(j)}+\bar{p}_{0}^{(j)} v+i \sum_{n \neq 0} e^{-i n v} \bar{p}_{n}^{(j)} / n, \quad j=1,2
\end{aligned}
$$

From Eq.2.9 we see that the periodicity properties of the $A$ and $B$ fields can be parametrized by the zero mode momenta e.g. of $\vartheta_{1}$ :

$$
A(u+2 \pi)=e^{-4 \pi p_{0}^{(1)} \sqrt{\gamma}} A(u), \quad B(v+2 \pi)=e^{+4 \pi \bar{p}_{0}^{(1)} \sqrt{\gamma}} B(v)
$$

The complete symmetry of the treatment of the theory under the exchange of $\vartheta_{1}, \bar{\vartheta}_{1}$ and $\vartheta_{2}, \bar{\vartheta}_{2}$ even on the quantum level is the hallmark of Gervais-Neveu quantization

\footnotetext{
${ }^{2}$ Actually the situation is somewhat more subtle for singular solutions, as we will see later.

${ }^{3}$ Compared to ref. [25], we have changed the notation by replacing $\phi_{1} \rightarrow \vartheta_{1}, \phi_{2} \rightarrow \vartheta_{2},-\bar{\phi}_{1} \rightarrow$ $\bar{\vartheta}_{2},-\bar{\phi}_{2} \rightarrow \bar{\vartheta}_{1}$.
} 
and guarantees the preservation of the residual symmetry Eq.2.6. From Eqs.2.4, 2.7 we have that the fields $f_{m}^{(J)}$ can be written as products of exponentials of the $\vartheta_{1}$ and $\vartheta_{2}$ fields:

$$
f_{m}^{(J)}=e^{(J-m) \sqrt{\gamma} \vartheta_{1}} e^{(J+m) \sqrt{\gamma} \vartheta_{2}}
$$

Though this form is, in principle, accessible directly to quantization for any $J$ and $m$ (cf. ref. 12 ), for the purposes of the present analysis it is more appropriate to work with an alternative Coulomb-gas-type representation in terms of one free field only. Let us consider the special cases $m= \pm J$ of Eq.2.11 where we have

$$
f_{-J}^{(J)}=\left(\frac{1}{\sqrt{A^{\prime}}}\right)^{2 J}=e^{2 J \sqrt{\gamma} \vartheta_{1}}, \quad f_{J}^{(J)}=\left(\frac{A}{\sqrt{A^{\prime}}}\right)^{2 J}=e^{2 J \sqrt{\gamma} \vartheta_{2}},
$$

Using the periodicity requirement Eq.2.10, one easily derives the relations

$$
\begin{aligned}
& A(u)=\left\{e^{-4 \pi p_{0}^{(1)} \sqrt{\gamma}} \int_{0}^{u} f_{1}^{(-1)}(\rho) d \rho+\int_{u}^{2 \pi} f_{1}^{(-1)}(\rho) d \rho\right\} /\left(e^{-4 \pi p_{0}^{(1)} \sqrt{\gamma}}-1\right) \\
& \frac{-1}{A(u)}=\left\{e^{-4 \pi p_{0}^{(2)} \sqrt{\gamma}} \int_{0}^{u} f_{-1}^{(-1)}(\rho) d \rho+\int_{u}^{2 \pi} f_{-1}^{(-1)}(\rho) d \rho\right\} /\left(e^{-4 \pi p_{0}^{(2)} \sqrt{\gamma}}-1\right)
\end{aligned}
$$

Then we may rewrite Eq.2.4 as

$$
f_{m}^{(J)}=\sqrt{\left(\begin{array}{c}
2 J \\
J+m
\end{array}\right)} f_{-J}^{(J)} A^{J+m}=\sqrt{\left(\begin{array}{c}
2 J \\
J+m
\end{array}\right)} f_{J}^{(J)}\left(A^{-1}\right)^{J-m}
$$

The starting point of the quantization is to replace Eqs.2.8 by their quantum counterparts, so that we now have

$$
\left[\vartheta_{1}^{\prime}\left(\sigma_{1}\right), \vartheta_{1}^{\prime}\left(\sigma_{2}\right)\right]=\left[\vartheta_{2}^{\prime}\left(\sigma_{1}\right), \vartheta_{2}^{\prime}\left(\sigma_{2}\right)\right]=2 \pi i \delta^{\prime}\left(\sigma_{1}-\sigma_{2}\right)
$$

It was shown in ref. 19 that $\vartheta_{1}$ and $\vartheta_{2}$ are related by a complicated canonical transformation; however, the relation between the zero modes is simple:

$$
p_{0}^{(1)}=-p_{0}^{(2)} \quad, \quad \bar{p}_{0}^{(1)}=-\bar{p}_{0}^{(2)} .
$$

Instead of $p_{0}^{(1)}$ (or $p_{0}^{(2)}$ ) it will be more convenient to work with the rescaled zero mode

$$
\varpi:=i p_{0}^{(1)} \sqrt{\frac{2 \pi}{h}}
$$

with $h$ defined in terms of the central charge $C$ by

$$
h=\frac{\pi}{12}(C-13-\sqrt{(C-25)(C-1)})
$$

The parameter $h$ which is the deformation parameter of $\operatorname{sl}(2)$, is also in effect the Planck constant of the quantum Liouville theory. 


\section{The case of a single screening charge}

\subsection{The braiding of the holomorphic components}

Starting from the representation Eq.2.15, and following the method of ref. 12], we construct the quantum equivalents of the fields $f_{m}^{(J)}$. In the original work, they have been noted $V_{m}^{(J)}, \widetilde{V}_{m}^{(J)}$, or $U_{m}^{(J)}$, depending upon the normalization chosen. They are periodic up to a multiplicative constant and thus can be considered as Bloch waves. On the other hand, there is also a second basis of chiral operators $\xi_{M}^{(J)}$, which are by construction explicitly covariant under the quantum group [2] [9], and related to the Bloch wave fields by a linear transformation. In the present article, we will concentrate on the Bloch wave basis, except for the last section. We first explain the construction of the Bloch wave vertex operators and their exchange algebra, in part as a preparation for the case of two screening charges. The presentation follows ref.[26]. As discussed above, we consider the semi-infinite families of Bloch wave operators with $J+m$ or $J-m$ a non-negative integer. It turns out that there exists a consistent operator algebra where the two types of families do not mix. Thus we may concentrate on one type, say the case with $J+m=0,1, \ldots$ Then the quantum version of $f_{m}^{(J)}$ is most easily obtained from the quantum versions of (the left equality in) Eq.2.15, and of Eq.2.13. According to Eq.2.12, this leads to quantum expressions in terms of $\vartheta_{1}$ - note that the other case $(J-m$ integer) may be obtained by the replacement $\vartheta_{1} \leftrightarrow \vartheta_{2}$ everywhere (cf. also section 4.1.5).

To begin with, the factor $f_{-J}^{(J)}$ is replaced by the normal-ordered exponential

$$
\left.f_{-J}^{(J)}\right|_{\mathrm{qu}} \equiv U_{-J}^{(J)}=: e^{2 J \sqrt{h / 2 \pi} \vartheta_{1}}:
$$

The parameter $h$ is that of Eq.2.19. The change of the coefficient in the exponential is such that this field has conformal weight

$$
\Delta_{J}=-J-\frac{h}{\pi} J(J+1)
$$

If $2 J$ is a positive integer, this coincides with Kac's formula, and $U_{-J}^{(J)}$ is a $(1,2 J+1)$ primary in the BPZ classification. Now let us turn to the second factor $A^{J+m}$ appearing on the left equation of Eq.2.15. The classical expression Eq.2.13 for $A$ has a rather simple quantum generalization, which we will denote by $S$ to signify that it is is a primary field of dimension zero ("screening operator"), namely [21]

$$
S(\sigma)=e^{2 i h(\varpi+1)} \int_{0}^{\sigma} d \rho U_{1}^{(-1)}(\rho)+\int_{\sigma}^{2 \pi} d \rho U_{1}^{(-1)}(\rho)
$$

Apart from an overall change of normalization - removal of the denominator - and the introduction of normal orderings, the only change consists in the replacement $\varpi \rightarrow \varpi+1$ in the prefactor of the first integral. The quantum formula is such that $S$ is periodic up to a multiplicative factor

$$
S(\sigma+2 \pi)=e^{2 i h(\varpi+1)} S(\sigma) .
$$


This is the quantum version of the left equation in Eq.2.10. $S$ is the operator formed from the semiclassical screening charge

$$
\alpha_{-} \equiv 2 \sqrt{\frac{h}{2 \pi}}
$$

which tends to zero in the classical limit $h \rightarrow 0$, or $C \rightarrow \infty$ (cf. Eq.2.19). $\alpha_{-}$is just the same quantity that appears e.g. in the treatment of minimal models 32, except that there the central charge is smaller than one. The basic primary field of the Coulomb gas picture is now defined as

$$
U_{m}^{(J)}(\sigma)=U_{-J}^{(J)}(\sigma)[S(\sigma)]^{J+m}
$$

which is the quantum version of the first equality in Eq.2.15. The product of operators at the same point implied in Eq.3.6 exists for small enough $h$ (more on this below). Since $S$ is a screening operator, the conformal dimension of $U_{m}^{(J)}$ agrees with Eq.3.2. It should be emphasized that the Coulomb gas picture obtained here differs from the standard one 32] in that it is "operatorial", i.e. there are no contours which depend on the particular correlation function under consideration. It is easy to verify that

$$
U_{m}^{(J)} \varpi=(\varpi+2 m) U_{m}^{(J)}
$$

Here we are assuming $\varpi$ to be real, as is appropriate in the socalled elliptic sector of the theory (cf. section 4.1). Also in the rest of the review we will concentrate on this case, if not indicated otherwise. It is the case which appears to be directly related to (tree level) amplitudes in $c \leq 1$ string theory [7]. The normalization of the $U_{m}^{(J)}$ operators is given by

$$
<\varpi\left|U_{m}^{(J)}\right| \varpi+2 m>=I_{m}^{(J)}(\varpi)
$$

and $I_{m}^{(J)}(\varpi)$ can be computed to be 26 t

$$
\begin{gathered}
I_{m}^{(J)}(\varpi)=\left(2 \pi \Gamma\left(1+\frac{h}{\pi}\right)\right)^{J+m} e^{i h(J+m)(\varpi-J+m)} \\
\prod_{\ell=1}^{J+m} \frac{\Gamma[1+(2 J-\ell+1) h / \pi]}{\Gamma[1+\ell h / \pi] \Gamma[1-(\varpi+2 m-\ell) h / \pi] \Gamma[1+(\varpi+\ell) h / \pi]} .
\end{gathered}
$$

This formula illustrates an important point to be made about the integral representation Eq.3.6. For small enough $h$, the arguments of the gamma functions are all positive, and this corresponds to the domain where the integral representation is convergent. When $h$ increases, divergences appear. However, Eq.3.9 continues to make sense beyond the poles by the usual analytic continuation of the Gamma function. As is well known 11], the continuation of the ground state expectation value $I_{m}^{(J)}(\varpi)$ defines the continuation of the operator $U_{m}^{(J)}$ itself. Thus, $U_{m}^{(J)}$ is related to

\footnotetext{
${ }^{4}$ see ref. 33 for a similar calculation, applied to the degenerate case
} 
the normalized operator $V_{m}^{(J)}$ with $\left\langle\varpi\left|V_{m}^{(J)}(\sigma=0)\right| \varpi+2 m\right\rangle=1$ (introduced already in ref. [8] for half-integer positive $J$ ) by

$$
U_{m}^{(J)}=I_{m}^{(J)}(\varpi) V_{m}^{(J)}
$$

Note that $U_{-J}^{(J)} \equiv V_{-J}^{(J)}$.

We now come to the braiding algebra of the fields $U_{m}^{(J)}$. For half-integer positive spin - the case corresponding to Kac's table - it is well known that the braiding of the $U_{m}^{(J)}$ or $V_{m}^{(J)}$ is essentially given by a q- $6 j$-symbol, and the explicit formulae were determined in ref. [8] (The general result is summarized in appendix A). In ref. [12], this result was extended to arbitrary $J$. We will recall some basic points of the derivation that will be useful later on. The braiding relation takes the form

$$
U_{m}^{(J)}(\sigma) U_{m}^{\left(J^{\prime}\right)}\left(\sigma^{\prime}\right)=\sum_{m_{1}, m_{2}} R_{U}\left(J, J^{\prime} ; \varpi\right)_{m m^{\prime}}^{m_{2} m_{1}} U_{m_{2}}^{\left(J^{\prime}\right)}\left(\sigma^{\prime}\right) U_{m_{1}}^{(J)}(\sigma) .
$$

We only deal with the case $2 \pi>\sigma^{\prime}>\sigma>0$ explicitly. The other cases are deduced from the present one, by using the periodicity properties of the $U_{m}^{(J)}$ fields, and the fact that the $R$-matrix for the other order of $\sigma$ and $\sigma^{\prime}$ must be just the inverse of the one in Eq.3.11. The sums extend over non-negative integer $J+m_{1}$ resp. $J^{\prime}+m_{2}$ with the condition

$$
m_{1}+m_{2}=m+m^{\prime}=: m_{12} .
$$

Since one considers the braiding at equal $\tau$ one can let $\tau=0$ once and for all. As there are no null-vector decoupling equations for continuous $J$, the derivation of Eq.3.11 relies exclusively on the free field techniques summarized in the previous section. The basic point of our argument is that the exchange of two $U_{m}^{(J)}$ operators can be mapped into an equivalent problem in one-dimensional quantum mechanics, and becomes just finite-dimensional linear algebra. In view of Eqs.3.3, 3.6, the essential observation is that one only needs the braiding relations of $U_{-J}^{(J)} \equiv V_{-J}^{(J)}$ operators which are normal ordered exponentials ("tachyon operators"). One has

$$
V_{-J}^{(J)}(\sigma) V_{-J^{\prime}}^{\left(J^{\prime}\right)}\left(\sigma^{\prime}\right)=e^{-i 2 J J^{\prime} h \epsilon\left(\sigma-\sigma^{\prime}\right)} V_{-J^{\prime}}^{\left(J^{\prime}\right)}\left(\sigma^{\prime}\right) V_{-J}^{(J)}(\sigma)
$$

where $\epsilon\left(\sigma-\sigma^{\prime}\right)$ is the sign of $\sigma-\sigma^{\prime}$. The fact that Eq.3.13 depends on the position only through the order of $\sigma$ and $\sigma^{\prime}$ is crucial for the following argument; it means that the problem is in a sense of a topological nature. Indeed, the results we obtain for the single screening case are in agreement with those obtained by an analysis of the braiding problem for the Liouville theory on the lattice [10], though the language is rather different, and $6 j$ symbols do not explicitly appear in ref. [10]. The first step of the analysis is to note that when commuting the tachyon operators in $U_{m^{\prime}}^{\left(J^{\prime}\right)}\left(\sigma^{\prime}\right)$ through those of $U_{m}^{(J)}(\sigma)$, one only encounters phase factors of the form $e^{ \pm i 2 \alpha \beta h}$ resp. $e^{ \pm 6 i \alpha \beta h}$, with $\alpha$ equal to $J$ or $-1, \beta$ equal to $J^{\prime}$ or -1 , since we take $\sigma, \sigma^{\prime} \in[0,2 \pi]$. Hence we are led to decompose the integrals defining the screening charges $\mathrm{S}$ into pieces which commute with each other and with $V_{-J}^{(J)}(\sigma), V_{-J^{\prime}}^{\left(J^{\prime}\right)}\left(\sigma^{\prime}\right)$ up to one of the above phase factors. We consider explicitly only the case $0<\sigma<\sigma^{\prime}<2 \pi$ and write

$$
S(\sigma)=S_{\sigma \sigma^{\prime}}+S_{\Delta}, \quad S\left(\sigma^{\prime}\right)=S_{\sigma \sigma^{\prime}}+k(\varpi) S_{\Delta} \equiv S_{\sigma \sigma^{\prime}}+\tilde{S}_{\Delta},
$$




$$
\begin{aligned}
S_{\sigma \sigma^{\prime}} & :=k(\varpi) \int_{0}^{\sigma} V_{1}^{(-1)}(\rho) d \rho+\int_{\sigma^{\prime}}^{2 \pi} V_{1}^{(-1)}(\rho) d \rho, \\
S_{\Delta} & :=\int_{\sigma}^{\sigma^{\prime}} V_{1}^{(-1)}(\rho) d \rho, \quad k(\varpi):=e^{2 i h(\varpi+1)}
\end{aligned}
$$

Using Eq.3.13, we then get the following simple algebra for $S_{\sigma \sigma^{\prime}}, S_{\Delta}, \tilde{S}_{\Delta}$ :

$$
S_{\sigma \sigma^{\prime}} S_{\Delta}=q^{-2} S_{\Delta} S_{\sigma \sigma^{\prime}}, \quad S_{\sigma \sigma^{\prime}} \tilde{S}_{\Delta}=q^{2} \tilde{S}_{\Delta} S_{\sigma \sigma^{\prime}}, \quad S_{\Delta} \tilde{S}_{\Delta}=q^{4} \tilde{S}_{\Delta} S_{\Delta}
$$

and their commutation properties with $V_{-J}^{(J)}(\sigma), V_{-J^{\prime}}^{\left(J^{\prime}\right)}\left(\sigma^{\prime}\right)$ are given by

$$
\begin{array}{ll}
V_{-J}^{(J)}(\sigma) S_{\sigma \sigma^{\prime}}=q^{-2 J} S_{\sigma \sigma^{\prime}} V_{-J}^{(J)}(\sigma), & V_{-J^{\prime}}^{\left(J^{\prime}\right)}\left(\sigma^{\prime}\right) S_{\sigma \sigma^{\prime}}=q^{-2 J^{\prime}} S_{\sigma \sigma^{\prime}} V_{-J^{\prime}}^{\left(J^{\prime}\right)}\left(\sigma^{\prime}\right), \\
V_{-J}^{(J)}(\sigma) S_{\Delta}=q^{-2 J} S_{\Delta} V_{-J}^{(J)}(\sigma), & V_{-J}^{(J)}(\sigma) \tilde{S}_{\Delta}=q^{-6 J} \tilde{S}_{\Delta} V_{-J}^{(J)}(\sigma), \\
V_{-J^{\prime}}^{\left(J^{\prime}\right)}\left(\sigma^{\prime}\right) S_{\Delta}=q^{2 J^{\prime}} S_{\Delta} V_{-J^{\prime}}^{\left(J^{\prime}\right)}\left(\sigma^{\prime}\right), & V_{-J^{\prime}}^{\left(J^{\prime}\right)}\left(\sigma^{\prime}\right) \tilde{S}_{\Delta}=q^{-2 J^{\prime}} \tilde{S}_{\Delta} V_{-J^{\prime}}^{\left(J^{\prime}\right)}\left(\sigma^{\prime}\right) .
\end{array}
$$

Finally, all three screening pieces obviously shift the zero mode in the same way:

$$
\left.\begin{array}{c}
S_{\sigma \sigma^{\prime}} \\
S_{\Delta} \\
\tilde{S}_{\Delta}
\end{array}\right\} \varpi=(\varpi+2)\left\{\begin{array}{c}
S_{\sigma \sigma^{\prime}} \\
S_{\Delta} \\
\tilde{S}_{\Delta}
\end{array} .\right.
$$

Using Eqs.3.16 we can commute $\widetilde{V}_{-J}^{(J)}(\sigma)$ and $\tilde{V}_{-J^{\prime}}^{\left(J^{\prime}\right)}\left(\sigma^{\prime}\right)$ to the left on both sides of Eq.3.11, so that they can be cancelled. Then we are left with

$$
\begin{gathered}
\left(q^{-2 J^{\prime}} S_{\Delta}+q^{2 J^{\prime}} S_{\sigma \sigma^{\prime}}\right)^{J+m}\left(\tilde{S}_{\Delta}+S_{\sigma \sigma^{\prime}}\right)^{J^{\prime}+m^{\prime}} q^{2 J J^{\prime}}= \\
\sum_{m_{1}, m_{2}} R\left(J, J^{\prime} ; \varpi+2\left(J+J^{\prime}\right)\right)_{m m^{\prime}}^{m_{2} m_{1}}\left(q^{2 J} S_{\sigma \sigma^{\prime}}+q^{6 J} \tilde{S}_{\Delta}\right)^{J^{\prime}+m_{2}}\left(S_{\sigma \sigma^{\prime}}+S_{\Delta}\right)^{J+m_{1}}
\end{gathered}
$$

It is apparent from this equation that the braiding problem of the $U_{m}^{(J)}$ operators is governed by the Heisenberg-like algebra Eq.3.15, characteristic of one-dimensional quantum mechanics. We will proceed using the following simple representation of the algebra Eq.3.15 in terms of one-dimensional quantum mechanics ( $y$ and $y^{\prime}$ are arbitrary complex numbers):

$$
S_{\sigma \sigma^{\prime}}=y^{\prime} e^{2 Q}, \quad S_{\Delta}=y e^{2 Q-P}, \quad \tilde{S}_{\Delta}=y e^{2 Q+P}, \quad[Q, P]=i h .
$$

The third relation in Eq.3.19 follows from the second one in view of $\tilde{S}_{\Delta}=k(\varpi) S_{\Delta}$ (cf. Eq.3.14). This means we are identifying here $P \equiv i h \varpi$ with the zero mode of the original problem. Using $e^{2 Q+c P}=e^{c P} e^{2 Q} q^{c}$ we can commute all factors $e^{2 Q}$ to the right on both sides of Eq.3.18 and then cancel them. This leaves us with

$$
\begin{gathered}
q^{2 J J^{\prime}} \prod_{s=1}^{J+m}\left(y^{\prime} q^{2 J^{\prime}}+y q^{-(\varpi-2 J+2 s-1)}\right) \prod_{t=1}^{J^{\prime}+m^{\prime}}\left(y^{\prime}+y q^{\varpi-2 J^{\prime}+2 m+2 t-1}\right)= \\
\sum_{m_{1}} R_{U}\left(J, J^{\prime} ; \varpi\right)_{m}^{m_{2} m_{1}} \prod_{t=1}^{J^{\prime}+m_{2}}\left(y^{\prime} q^{2 J}+y q^{\varpi+4 J-2 J^{\prime}+2 t-1}\right) \prod_{s=1}^{J+m_{1}}\left(y^{\prime}+y q^{-\left(\varpi-2 J+2 m_{2}+2 s-1\right)}\right)
\end{gathered}
$$


where we have shifted back $\varpi+2\left(J+J^{\prime}\right) \rightarrow \varpi$ compared to Eq.3.18. Since the overall scaling $y \rightarrow \lambda y, y^{\prime} \rightarrow \lambda y^{\prime}$ only gives back Eq.3.12, we can set $y^{\prime}=1$.

The solution of these equations, which was derived in ref. 12], will be cast under the convenient form

$$
R_{U}\left(J, J^{\prime}, \varpi\right)_{m m^{\prime}}^{m_{2} m_{1}}=e^{-i \pi\left(\Delta_{c}+\Delta_{b}-\Delta_{e}-\Delta_{f}\right)} \frac{\kappa_{a b}^{e} \kappa_{d e}^{c}}{\kappa_{d b}^{f} \kappa_{a f}^{c}}\left\{\begin{array}{l|l|l}
a & b & e \\
d & c & f
\end{array}\right\}
$$

where $\left\{\begin{array}{ll|l}a & b & e \\ d & c & f\end{array}\right\}$ is the q-6j-symbol generalized to continous spins, with arguments

$$
\begin{gathered}
a=J, \quad b=x+m+m^{\prime}, \quad c=x \equiv\left(\varpi-\varpi_{0}\right) / 2 \\
d=J^{\prime}, \quad e=x+m_{2}, \quad f=x+m,
\end{gathered}
$$

and the coefficients $\kappa_{J_{1} J_{2}}^{J_{12}}$ are given by

$$
\begin{gathered}
\kappa_{J_{1} J_{2}}^{J_{12}}=\left(\frac{h e^{-i(h+\pi)}}{2 \pi \Gamma(1+h / \pi) \sin h}\right)^{J_{1}+J_{2}-J_{12}} e^{i h\left(J_{1}+J_{2}-J_{12}\right)\left(J_{1}-J_{2}-J_{12}\right)} \times \\
\\
\prod_{k=1}^{J_{1}+J_{2}-J_{12}} \sqrt{\frac{\left\lfloor 1+2 J_{1}-k\right\rfloor}{\lfloor k\rfloor\left\lfloor 1+2 J_{2}-k\right\rfloor\left\lfloor-\left(1+2 J_{12}+k\right)\right\rfloor}} .
\end{gathered}
$$

Recall that we let $\lfloor x\rfloor=\sin (h x) / \sin h$ in general. The last equation makes sense for arbitrary $J_{1}, J_{2}, J_{12}$ such that $J_{1}+J_{2}-J_{12}$ is a non-negative integer. The r.h.s.of Eq.3.21 may be expressed in terms of q-hypergeometric functions by the formula

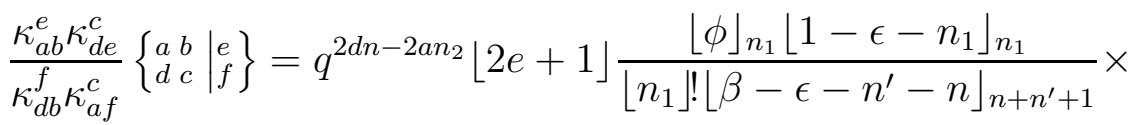

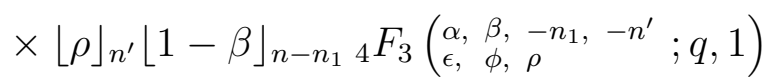

with

$$
\begin{gathered}
\alpha=-a-c+f, \quad \beta=-c-d+e, \\
n_{1}=a+b-e, \quad n_{2}=d+e-c, \quad n=f+a-c \quad n^{\prime}=b+d-f ; \\
\epsilon=-(a+b+c+d+1), \quad \phi=1+n-n_{1}, \quad \rho=e+f-a-d+1 .
\end{gathered}
$$

Here we have defined

$$
\begin{gathered}
{ }_{4} F_{3}\left(\begin{array}{l}
a, b, c, d \\
e, f, g
\end{array} ; q, \rho\right)=\sum_{n=0}^{\infty} \frac{\lfloor a\rfloor_{n}\lfloor b\rfloor_{n}\lfloor c\rfloor_{n}\lfloor d\rfloor_{n}}{\lfloor e\rfloor_{n}\lfloor f\rfloor_{n}\lfloor g\rfloor_{n}\lfloor n\rfloor !} \rho^{n}, \\
\lfloor a\rfloor_{n}:=\lfloor a\rfloor\lfloor a+1\rfloor \cdots\lfloor a+n-1\rfloor, \quad\lfloor a\rfloor_{0}:=1 .
\end{gathered}
$$

In Eq.3.24, the prefactor involves products of the type just recalled with indices

$$
n_{1}=J+m_{1}, \quad n_{2}=J+m_{2}, \quad n=J+m, \quad n^{\prime}=J+m^{\prime} .
$$

${ }^{5}$ We follow the prescription of refs. [7], [17] for the definition of the square roots (cf. also below Eq.3.31). In particular, the square root in Eq.3.23 is to be understood as the product resp. quotient of the square roots of the individual factors consisting of a single q-number. 
Since they are equal to the screening numbers, they are positive integers. Thus Eq.3.24 makes sense for arbitrary spins provided the screening numbers are integers, and is the appropriate generalization.

The method used to derive Eq.3.21 was to transform the hypergeometric function into another one such that the desired relations Eq. 3.20 follow from the orthogonality relation of the associated Askey-Wilson polynomials. In this connection, let us note that a simple reshuffling of the parameters of the latter form allows to verify that the usual orthogonality relations of the 6 -j symbols extend to our case. One has, in general,

$$
\sum_{J_{23}}\left\{\begin{array}{ll|l}
J_{1} & J_{2} & J_{12} \\
J_{3} & J_{123} & J_{23}
\end{array}\right\}\left\{\begin{array}{ll|l}
J_{1} & J_{2} \\
J_{3} & J_{123} & K_{12} \\
J_{23}
\end{array}\right\}=\delta_{J_{12}-K_{12}}
$$

where the $J$ 's are arbitrary except for the constraint that the screening numbers

$$
\begin{gathered}
n_{1}=J_{1}+J_{2}-J_{12}, \quad n_{2}=J_{3}+J_{12}-J_{123}, \quad n=J_{1}+J_{23}-J_{123}, \quad n^{\prime}=J_{2}+J_{3}-J_{23}, \\
\tilde{n}_{1}=J_{1}+J_{2}-K_{12}, \quad \tilde{n}_{2}=J_{3}+K_{12}-J_{123},
\end{gathered}
$$

are positive integers. These conditions fix the range of summation over $J_{23}$.

The basis $U_{m}^{(J)}$, apart from its manageability, has another practical virtue: Its braiding (and fusion) properties are given in a form which involves no square roots, but only (q-deformed) rational functions, and no phase ambiguities can arise. On the other hand, from the quantum group point of view it is more natural to consider a basis where the braiding (and fusion) is given exclusively in terms of the $6 j$-symbol (the latter does however involve square roots). For this purpose, the authors of ref. [8] introduced the fields $\widetilde{V}_{m}^{(J)}$, which are defined by

$$
\tilde{V}_{m}^{(J)}=g_{J, x+m}^{x} V_{m}^{(J)}
$$

As before we let $x=\left(\varpi-\varpi_{0}\right) / 2$. The coupling constants $g$ are defined by

$$
\begin{gathered}
g_{J, x+m}^{x}=\left(\frac{h}{\pi}\right)^{J+m} \prod_{k=1}^{J+m} \sqrt{F[1+(2 J-k+1) h / \pi]} \sqrt{F[(\varpi+2 m-k) h / \pi]} \times \\
\prod_{k=1}^{J+m} \sqrt{F[-(\varpi+k) h / \pi]} / \sqrt{F[1+k h / \pi]}
\end{gathered}
$$

where, as usual, $F(z):=\Gamma(z) / \Gamma(1-z)$. The treatment of the square roots requires some care. We follow the prescription of ref.[7] also used in ref. [17]. Eq.33.31 immediately extends to the case of non integer $J$, as $J+m$ remains a positive integer. We then have $\left(m_{12}:=m_{1}+m_{2}=m+m^{\prime}\right)$

$$
\begin{gathered}
\tilde{V}_{m}^{(J)}(\sigma) \widetilde{V}_{m^{\prime}}^{\left(J^{\prime}\right)}\left(\sigma^{\prime}\right)=e^{-i \pi\left(\Delta_{x}+\Delta_{x+m_{12}}-\Delta_{x+m}-\Delta_{x+m_{2}}\right)} \times \\
\sum_{m_{1}, m_{2}}\left\{\begin{array}{ll|l}
J & \begin{array}{l}
J_{J^{\prime}} \\
x+m_{12}
\end{array} & \begin{array}{l}
x+m_{2} \\
x+m
\end{array}
\end{array}\right\} \widetilde{V}_{m_{2}}^{\left(J^{\prime}\right)}\left(\sigma^{\prime}\right) \widetilde{V}_{m_{1}}^{(J)}(\sigma)
\end{gathered}
$$

\footnotetext{
${ }^{6}$ This transformation formula is derived in ref. 17.
} 
The relation with $U_{m}^{(J)}$ is given by

$$
U_{m}^{(J)}=\frac{I_{m}^{(J)}(\varpi)}{g_{J, x+m}^{x}} \widetilde{V}_{m}^{(J)} \equiv \frac{1}{\kappa_{J, x+m}^{x}} \widetilde{V}_{m}^{(J)} .
$$

The $\kappa$ coefficient is of course given by Eq.3.23. Concerning the right-moving modes, the braiding algebra is given by

$$
\begin{gathered}
\overline{\widetilde{V}}_{m}^{(J)}(\sigma) \overline{\widetilde{V}}_{m^{\prime}}^{\left(J^{\prime}\right)}\left(\sigma^{\prime}\right)=e^{i \pi\left(\Delta_{\bar{x}}+\Delta_{\bar{x}+m_{12}}-\Delta_{\bar{x}+m}-\Delta_{\bar{x}+m_{2}}\right)} \times \\
\sum_{m_{1}, m_{2}}\left\{\begin{array}{ll|l}
J_{1} & \bar{x}+m_{12} & \bar{x}+m_{2} \\
J_{2} & \bar{x} & \bar{x}+m
\end{array}\right\} \overline{\widetilde{V}}_{m_{2}}^{\left(J^{\prime}\right)}\left(\sigma^{\prime}\right) \overline{\widetilde{V}}_{m_{1}}^{(J)}(\sigma)
\end{gathered}
$$

where we let $\bar{x}=\left(\bar{\varpi}-\varpi_{0}\right) / 2$. The only difference with Eq.3.32 is the change of sign of the phase factor. This may be verified by redoing the whole derivation. In refs. [21], [7] it was remarked that the right-mover braiding matrix is deduced from the left-mover one by changing $i=\sqrt{-1}$ into $-i$, since this correctly changes the orientation of the complex plane. This complex conjugation is most easily performed using the $U$ fields, since the braiding matrix Eq.3.21 is real apart from the first phase factor. For the $\widetilde{V}$ fields, there is a slight subtlety related again to the appearance of the redundant square roots in Eq.3.23. The correct rule is to take the same definition for the square roots for left and right movers. Thus the right-moving coupling constant $\bar{g}_{J, \bar{x}+m}^{\bar{x}}$ is given by the same expression Eq.3.31, not its complex conjugate. The same prescription should be followed for the roots appearing in $\bar{\kappa}_{J, \bar{x}+m}^{\bar{x}}$, while taking the usual complex conjugate for the phase factor appearing in front of the product in Eq.3.23. Note that $\varpi$ is always to be treated as real formally in this context, even in the hyperbolic sector where it is actually purely imaginary (cf. below).

\section{Solving of the Liouville quantum dynamics}

\subsection{The Liouville exponential}

First, let us note that, as $h$ is real in the weak-coupling regime, the hermiticity of energy-momentum allows for $\varpi, \bar{\varpi}$ real or purely imaginary, corresponding to the elliptic resp. hyperbolic sector of the theory [22] (see also [4] for the case of open boundary conditions). In the former case, which we consider in this review, we will see that the locality conditions are fulfilled if

$$
\varpi-\bar{\varpi}=k \pi / h, \quad k \in \mathbf{Z}
$$

Eq.4.1 has an immediate interpretation as the natural generalization of the classical boundary conditions 21]. Moreover we will show that the appropriate definition of the Liouville exponential for arbitrary $J$ is

$$
e^{-J \alpha_{-} \Phi(\sigma, \tau)}=\sum_{m=-J}^{\infty} \mu_{0}^{J+m} \widetilde{V}_{m}^{(J)}(u) \overline{\widetilde{V}}_{m}^{(J)}(v)
$$

where $\alpha_{-}=2 \sqrt{h / 2 \pi}$ is the screening charge. The constant $\mu_{0}^{J+m}$ will not be fixed by braiding or fusion. It will be determined below when we derive the field equations. 


\subsubsection{Locality}

Let us now check locality. In the approach of refs. [21] [7], one takes the zero modes of the left-moving and right-moving Liouville modes to commute, so that $U$ and $\bar{U}$ commute. However, operators involving both chiralities should be applied only to states fulfilling Eq.4.1, and conserve this condition. This is why we must have $\bar{m}=m$ in Eq.4.2. Next we observe that if Eq.4.1 is valid,

$$
\bar{R} \overline{\widetilde{V}}\left(J, J^{\prime} ; \bar{\varpi}\right)_{\bar{m} \bar{m}_{\bar{m}^{\prime}}}^{\bar{m}_{2} \bar{m}_{1}}=\bar{R} \overline{\widetilde{V}}\left(J, J^{\prime} ; \varpi\right)_{\bar{m} \bar{m}^{\prime}}^{\bar{m}_{2} \bar{m}_{1}}
$$

as can be verified easily. The same is true for $\bar{R}_{\bar{U}}$. Thus we have

$$
\begin{gathered}
\overline{\widetilde{V}}_{m}^{(J)}(\sigma) \overline{\widetilde{V}}_{m^{\prime}}^{\left(J^{\prime}\right)}\left(\sigma^{\prime}\right)=e^{+i \pi\left(\Delta_{x}+\Delta_{x+m_{12}}-\Delta_{x+m}-\Delta_{x+m_{2}}\right)} \times \\
\sum_{m_{1}, m_{2}}\left\{\begin{array}{rr|r}
J_{1} & x+m_{12} & \begin{array}{c}
x+m_{2} \\
J_{2}
\end{array} \\
x+m
\end{array}\right\} \overline{\widetilde{V}}_{m_{2}}^{\left(J^{\prime}\right)}\left(\sigma^{\prime}\right) \overline{\widetilde{V}}_{m_{1}}^{(J)}(\sigma)
\end{gathered}
$$

Then, according to Eqs.3.32, 4.4 we get

$$
\begin{gathered}
e^{-J \alpha_{-} \Phi(\sigma, \tau)} e^{-J^{\prime} \alpha_{-} \Phi\left(\sigma^{\prime}, \tau\right)}= \\
\sum_{m, m^{\prime} m_{1}, m_{2} ; \bar{m}_{1}, \bar{m}_{2}} e^{i h\left(\bar{m}_{2}-m_{2}\right)\left(\bar{m}_{2}-m_{2}+\varpi\right)}\left\{\begin{array}{ll}
J & x+m_{1}+m_{2} \\
J^{\prime} & x
\end{array} \mid \begin{array}{l}
x+m_{2} \\
x+m
\end{array}\right\}\left\{\begin{array}{ll}
J & x+m_{1}+m_{2} \\
J^{\prime} & x
\end{array} \mid \begin{array}{l}
x+\bar{m}_{2} \\
x+m
\end{array}\right\} \\
\times\left.\mu_{0}^{J+m+J^{\prime}+m^{\prime}} \widetilde{V}_{m_{2}}^{\left(J^{\prime}\right)}\left(u^{\prime}\right) \widetilde{V}_{m_{1}}^{(J)}(u) \overline{\widetilde{V}}_{m_{2}}^{\left(J^{\prime}\right)}\left(v^{\prime}\right) \overline{\widetilde{V}}_{m_{1}}^{(J)}(v)\right|_{\substack{m_{1}+m_{2}=m+m^{\prime} \\
\bar{m}_{1}+\bar{m}_{2}=m+m^{\prime}}},
\end{gathered}
$$

One first sums over $m$, with fixed $m_{12}=m+m^{\prime}$. This precisely corresponds to the summation over $J_{23}$ in Eq.3.29. Thus only $m_{2}=\bar{m}_{2}$ contributes. This gives immediately

$$
e^{-J_{1} \alpha_{-} \Phi\left(\sigma_{1}, \tau\right)} e^{-J_{2} \alpha_{-} \Phi\left(\sigma_{2}, \tau\right)}=e^{-J_{2} \alpha_{-} \Phi\left(\sigma_{2}, \tau\right)} e^{-J_{1} \alpha_{-} \Phi\left(\sigma_{1}, \tau\right)},
$$

and the Liouville exponential is local for arbitrary $J$. We remark that Eq.4.1 is not only sufficient, but also necessary for locality, as was observed in ref. 21] for the special case $J=1 / 2$.

\subsubsection{Closure by fusion}

In the preceding analysis, we have discussed only the braiding properties of the chiral fields resp. the Liouville exponentials. However, according to the general Moore-Seiberg formalism[15], fusion (in the sense of the full operator product) and braiding are not independent. Assuming the validity of the Moore-Seiberg relation between fusion and braiding matrix, we then obtain immediately that the fusion of the $\widetilde{V}$ fields should be given by (cf. also ref.[17])

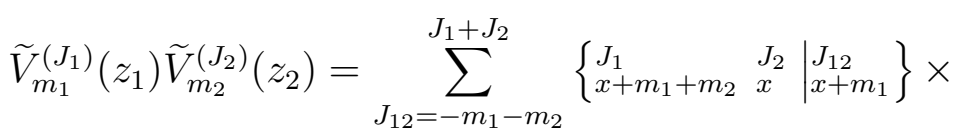

\footnotetext{
${ }^{7}$ The situation is quite different in the strong coupling theory, see e.g. ref. 117 .
} 


$$
\sum_{\left\{\nu_{12}\right\}} \tilde{V}_{m_{1}+m_{2}}^{\left(J_{12},\left\{\nu_{12}\right\}\right)}\left(z_{2}\right)<\varpi_{J_{12}},\left\{\nu_{12}\right\}\left|\widetilde{V}_{J_{2}-J_{12}}^{\left(J_{1}\right)}\left(z_{1}-z_{2}\right)\right| \varpi_{J_{2}}>
$$

In Eq.4.7 we have changed variables by letting $z=e^{i(\tau+\sigma)}, \bar{z}=e^{i(\tau-\sigma)}$ (recall that we are using Minkowski world-sheet variables). The only difference to the positive half-integer spin case, which was completely analyzed in ref. $8 \|$, is that the $J_{12}$-sum now extends to $-m_{1}-m_{2}$ instead of $\left|J_{1}-J_{2}\right|$. Indeed, the positivity of the screening numbers appearing in the braiding matrix leads via the Moore-Seiberg relation to the positivity of the screening numbers $n_{1}=J_{1}+m_{1}, n_{2}=J_{2}+m_{2}, p_{1,2}=$ $J_{1}+J_{2}-J_{12}, n=J_{12}+m_{1}+m_{2}$ of the fusion matrix. In ref.[17], it has been shown that the generalized $6 j$-symbol of Eq.3.21, together with the positivity condition for the screening charges, fulfills all the necessary identities for the polynomial equations to be valid with continous spins. This provides a strong argument that the fusion matrix of Eq.4.7 is indeed the correct one, even though we have not attempted to derive it directly as we did for the braiding. Making use of the analogous equation for the bar components, one sees that the operator-product expansion of Liouville exponentials may be written as

$$
\begin{aligned}
& e^{-J_{1} \alpha_{-} \Phi\left(z_{1}, \bar{z}_{1}\right)} e^{-J_{2} \alpha_{-} \Phi\left(z_{2}, \bar{z}_{2}\right)}= \\
& \sum_{m_{1}, m_{2}} \sum_{J_{12}, \bar{J}_{12}}\left\{\begin{array}{ll|l}
J_{1} & J_{2} & J_{12} \\
x+m_{1}+m_{2} & x & x+m_{1}
\end{array}\right\}\left\{\begin{array}{ll|l}
J_{1} & J_{2} & \bar{J}_{12} \\
\bar{x}+m_{1}+m_{2} & \bar{x} & \bar{x}+m_{1}
\end{array}\right\} \times \\
& \mu_{0}^{J_{1}+m_{1}+J_{2}+m_{2}} \sum_{\left\{\nu_{12}\right\},\left\{\bar{\nu}_{12}\right\}} \widetilde{V}_{m_{1}+m_{2}}^{\left(J_{12},\left\{\nu_{12}\right\}\right)}\left(z_{2}\right) \overline{\widetilde{V}}_{m_{1}+m_{2}}^{\left(\bar{J}_{12},\left\{\bar{\nu}_{12}\right\}\right)}\left(\bar{z}_{2}\right) \times \\
& <\varpi_{J_{12}},\left\{\nu_{12}\right\}\left|\widetilde{V}_{J_{2}-J_{12}}^{\left(J_{1}\right)}\left(z_{1}-z_{2}\right)\right| \varpi_{J_{2}}><\bar{\varpi}_{\bar{J}_{12}},\left\{\bar{\nu}_{12}\right\}\left|\widetilde{\widetilde{V}}_{J_{2}-\bar{J}_{12}}^{\left(J_{1}\right)}\left(\bar{z}_{1}-\bar{z}_{2}\right)\right| \bar{\varpi}_{J_{2}}>
\end{aligned}
$$

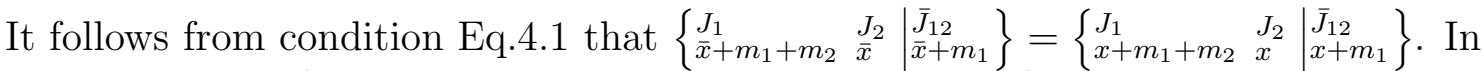
the same way as for locality, the summation over $m_{1}$ with fixed $m_{1}+m_{2}$ then reduces to the orthogonality relation for $6 j$-symbols so that only $J_{12}=\bar{J}_{12}$ contributes, and one gets

$$
\begin{gathered}
e^{-J_{1} \alpha_{-} \Phi\left(z_{1}, \bar{z}_{1}\right)} e^{-J_{2} \alpha_{-} \Phi\left(z_{2}, \bar{z}_{2}\right)}= \\
\sum_{m, J_{12}} \sum_{\left\{\nu_{12}\right\},\left\{\bar{\nu}_{12}\right\}} \mu_{0}^{J_{12}+m} \widetilde{V}_{m}^{\left(J_{12},\left\{\nu_{12}\right\}\right)}\left(z_{2}\right) \overline{\widetilde{V}}_{m}^{\left(J_{12},\left\{\bar{\nu}_{12}\right\}\right)}\left(\bar{z}_{2}\right) \mu_{0}^{J_{1}+J_{2}-J_{12}} \times \\
<\varpi_{J_{12}},\left\{\nu_{12}\right\}\left|\widetilde{V}_{J_{2}-J_{12}}^{\left(J_{1}\right)}\left(z_{1}-z_{2}\right)\right| \varpi_{J_{2}}><\bar{\varpi}_{J_{12}},\left\{\bar{\nu}_{12}\right\}\left|\overline{\widetilde{V}}_{J_{2}-J_{12}}^{\left(J_{1}\right)}\left(\bar{z}_{1}-\bar{z}_{2}\right)\right| \bar{\varpi}_{J_{2}}>.
\end{gathered}
$$

The second line clearly involves the descendants of the Liouville exponentials which we denote by

$$
e^{-J \alpha_{-} \Phi^{\{\nu\},\{\bar{\nu}\}}(z, \bar{z})} \equiv \sum_{m} \mu_{0}^{J+m} \widetilde{V}_{m}^{\left(J_{12},\{\nu\}\right)}(z) \overline{\widetilde{V}}_{m}^{\left(J_{12},\{\bar{\nu}\}\right)}(\bar{z})
$$

As regards the last line, it is simply the corresponding matrix element of the Liouville exponential. One finally gets

$$
e^{-J_{1} \alpha_{-} \Phi\left(z_{1}, \bar{z}_{1}\right)} e^{-J_{2} \alpha_{-} \Phi\left(z_{2}, \bar{z}_{2}\right)}=\sum_{J_{12}=-m_{1}-m_{2}}^{J_{1}+J_{2}} \sum_{\{\nu\},\{\bar{\nu}\}} e^{-J_{12} \alpha_{-} \Phi^{\{\nu\},\{\bar{\nu}\}}\left(z_{2}, \bar{z}_{2}\right)_{\times}}
$$




$$
<\varpi_{J_{12}}, \bar{\varpi}_{J_{12}} ;\{\nu\},\{\bar{\nu}\}\left|e^{-J_{1} \alpha_{-} \Phi\left(z_{1}-z_{2}, \bar{z}_{1}-\bar{z}_{2}\right)}\right|_{\varpi_{J_{2}}, \bar{\varpi}_{J_{2}}>} .
$$

The notation for the matrix element should be self-explanatory 8 . One sees that the Liouville exponential is closed by fusion for arbitary $J$ to all orders in the descendants.

\subsubsection{The cosmological constant revisited.}

The braiding relation is invariant under the transformation

$$
\exp \left(-J \alpha_{-} \Phi(z, \bar{z})\right) \rightarrow T \exp \left(-J \alpha_{-} \Phi(z, \bar{z})\right) T^{-1},
$$

where $T$ is an arbitrary function of the zero modes $\varpi$ and $\bar{\varpi}$. This is why locality does not completely determine the Liouville exponentials. We have discussed this point in detail in ref.25. Concerning the fusion equation, the transformation just considered does not act on the last term on the right-hand side which is a c-number. The definition Eq.4.2 we have chosen is such that this term - a compact bookkeeping device to handle all the descendants - is precisely given by the matrix elements of the Liouville exponential itself, without any additional normalization factor. It is thus quite natural. The only remaining ambiguity is the arbitrariness in $\mu_{0}$. Changing this parameter is tantamount to changing the cosmological constant following ref.[7]. Indeed, the fusing and braiding relations of the $\widetilde{V}$ fields are invariant if we make the change $\widetilde{V}_{m}^{(J)} \rightarrow \mu_{c}^{(J+m) / 2} \widetilde{V}_{m}^{(J)}$. Any such change is generated by a combination of a field redefinition $\left(\alpha_{-} \Phi \rightarrow \alpha_{-} \Phi-\ln \mu_{c}\right)$ and a similarity transformation of the form Eq.4.12. Thus the most general field satisfying Eqs.4.6 and 4.11 is given by

$$
e_{\mu_{c}}^{-J \alpha_{-} \Phi(\sigma, \tau)}=\sum_{m=-J}^{\infty}\left(\mu_{0} \mu_{c}\right)^{J+m} \widetilde{V}_{m}^{(J)}(u) \overline{\widetilde{V}}_{m}^{(J)}(v)=\mu_{c}^{J} \mu_{c}^{-\varpi / 2} e^{-J \alpha_{-} \Phi(\sigma, \tau)} \mu_{c}^{\varpi / 2}
$$

We will determine $\mu_{0}$ below so that it corresponds to a cosmological constant equal to one.

\subsubsection{Expression in terms of Coulomb-gas fields}

According to Eqs.3.6 and 3.33, Eq.4.2 may be rewritten as

$$
e^{-J \alpha_{-} \Phi(\sigma, \tau)}=\sum_{m=-J}^{\infty} \mu_{0}^{J+m} \kappa_{J, x+m}^{x} \bar{\kappa}_{J, \bar{x}+m}^{\bar{x}} U_{m}^{(J)}(u) \bar{U}_{m}^{(J)}(v)
$$

It is easy to see using condition Eq.4.1 that

$$
\bar{\kappa}_{J, \bar{x}+m}^{\bar{x}}=\bar{\kappa}_{J, x+m}^{x}
$$

\footnotetext{
${ }^{8}$ It is implied here that charge conservation should be used for the evaluation of the matrix element, such that only the term appearing in Eq.4.9 survives. According to ref.[31], charge conservation actually does not hold for the 3-point functions $\left\langle\varpi^{\prime}\left|e^{-J \alpha \Phi}(z)\right| \varpi\right\rangle$ with continous $J$. From this point of view, the notation of Eq.4.11 is of course not rigorously appropriate.
} 
Thus the square roots combine pairwise and we are left with a rational expression.

$$
\begin{gathered}
e^{-J \alpha_{-} \Phi(\sigma, \tau)=} \sum_{m=-J}^{\infty} \tilde{\mu}_{0}^{J+m}(-1)^{J+m} \prod_{k=1}^{J+m} \frac{\lfloor 1+2 J-k\rfloor}{\lfloor k\rfloor\lfloor\varpi+2 m-k\rfloor\lfloor\varpi+k\rfloor} \times \\
V_{-J}^{(J)}(u) \bar{V}_{-J}^{(J)}(v) S^{J+m} \bar{S}^{J+m}
\end{gathered}
$$

where we have let

$$
\tilde{\mu}_{0}=\mu_{0}\left(\frac{h}{2 \pi \Gamma(1+h / \pi) \sin h}\right)^{2}
$$

Using this Coulomb-gas expression, together with the mentioned orthogonality relations for Askey-Wilson polynomials, it is then possible to directly verify the locality of the Liouville exponential, without encountering any square root ambiguity. Note that Eq.4.15 depends only on $\varpi$, not on $\bar{\varpi}$. On the other hand, the analysis of ref. 21] for $J=1 / 2$ in the elliptic sector, when translated to the Coulomb gas basis, gives coefficients with an explicit dependence on $k$ of Eq.4.1. Nevertheless, the two forms are equivalent, as they must, by means of a basis transformation Eq.4.12, hence indistinguishable from the point of view of locality.

\subsection{5 $\vartheta_{1} \leftrightarrow \vartheta_{2}$ invariance}

In section 2 we noted the existence of a symmetry of the theory under the exchange of the two free fields $\vartheta_{1}$ under $\vartheta_{2}$, the residual symmetry remaining after fixing the $S L_{2}(\mathbf{C})$ invariance. On the other hand, on the quantum level the expressions we have derived in the present review for the Liouville field and its exponentials are not evidently symmetric under this exchange. However, we must remember here that the requirement of locality really fixed these operators only up to a similarity transformation Eq.4.12 (the particular form Eq.4.2 resp. Eq.4.15 was only distinguished by its simplicity and its natural behaviour under fusion). Thus a priori we can expect $\vartheta_{1} \leftrightarrow \vartheta_{2}$ invariance only to be valid up to a similarity transformation. As a matter of fact, we will show (for $J$ half-integer positive) that there exists a transformation $T(\varpi, \bar{\varpi})$ such that

$$
T(\varpi, \bar{\varpi}) e_{(1)}^{-J \alpha_{-} \Phi} T^{-1}(\varpi, \bar{\varpi})=T(-\varpi,-\bar{\varpi}) e_{(2)}^{-J \alpha_{-} \Phi} T^{-1}(-\varpi,-\bar{\varpi}) .
$$

where the index (1) resp. (2) indicates the use of the $\vartheta_{1}$ resp. $\vartheta_{2}$ representation. This shows in addition that it is possible to choose particular representatives in the equivalence class of fields defined by Eq.4.12 which are manifestly $\vartheta_{1} \leftrightarrow \vartheta_{2}$ symmetric. To prove this we first observe that $\vartheta_{1} \leftrightarrow \vartheta_{2}$ takes $\varpi$ into $-\varpi$ (cf. Eq.2.17), whereas the normalized operators $V_{m}^{(J)}$ behave as

$$
V_{m}^{(J)} \rightarrow V_{-m}^{(J)}
$$

(similarly for the right-movers). The latter follows by comparison of the conformal weights and zero mode shifts of the $V_{m}^{(J)}$ operators built from $\vartheta_{1}$ resp. $\vartheta_{2}$, as these two properties define normalized primary fields uniquely. For positive half-integer $J$, 
the summation range in Eq.4.15 is $m=-J, \ldots J$, hence symmetric under $m \rightarrow-m$, and the exchange $\vartheta_{1} \leftrightarrow \vartheta_{2}$ essentially amounts only to a reorganization of terms. Then after commuting the $T$ operators to the left or to the right on both sides, Eq.4.17 can be solved straightforwardly. A particular solution is

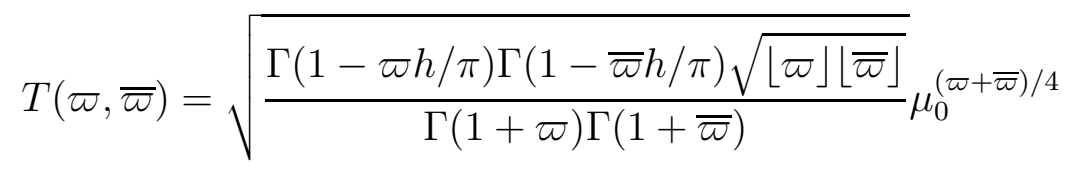

The last factor means effectively I $^{\text {that }}$ we should put $\mu_{0}=1$ in Eq.4.2 and Eq.4.15 (cf Eq.4.13), and so we will take $\mu_{0}=1$ in the following. The solution Eq.4.19 is unique up to the replacement $T(\varpi, \bar{\varpi}) \rightarrow T(\varpi, \bar{\varpi}) T_{1}(\varpi, \bar{\varpi})$, with

$$
\frac{T_{1}(\varpi, \bar{\varpi}) T_{1}(-\varpi-2 m,-\bar{\varpi}-2 m)}{T_{1}(\varpi+2 m, \bar{\varpi}+2 m) T_{1}(-\varpi,-\bar{\varpi})}=1
$$

Unfortunately the case of continous $J$, Eq.4.17 is not so easy to analyze, as the family of operators $V_{m}^{(J)}$ with $J+m=0,1,2, \ldots$ is no longer invariant under the replacement $m \rightarrow-m$, and Eq. 4.17 becomes highly nontrivial. We leave this problem for future work and will restrict also in the next subsection to the case of positive half-integer $J$.

\subsubsection{Hermiticity}

Another property of the Liouville exponentials that has not yet been discussed is hermiticity. As was worked out by Gervais and Neveu a long time ago 11, the free fields possess the following behaviour under hermitian conjugation (for brevity of notation we write only the left-movers explicitly):

$$
\begin{array}{lll}
\vartheta_{1}^{\dagger}=\vartheta_{1} \quad, \quad \vartheta_{2}^{\dagger}=\vartheta_{2} & \left(\varpi=-\varpi^{*}\right) \\
\vartheta_{1}^{\dagger}=\vartheta_{2} \quad, \quad \vartheta_{2}^{\dagger}=\vartheta_{1} & \left(\varpi=\varpi^{*}\right)
\end{array}
$$

The first case corresponds to the hyperbolic sector of the theory, the second to the elliptic sector which we consider here. Consequently, one has for the vertex operators resp. screening charges:

$$
\begin{gathered}
V_{-J}^{(J)^{\dagger}}=V_{-J}^{(J)}, V_{J}^{(J)^{\dagger}}=V_{J}^{(J)}, S_{(i)}^{\dagger}=S_{(i)} \quad\left(\varpi=-\varpi^{*}\right) \\
V_{-J}^{(J)}=V_{J}^{(J)}, V_{J}^{(J)}{ }^{\dagger}=V_{-J}^{(J)}, S_{(i)}^{\dagger}=S_{(i)} \quad\left(\varpi=\varpi^{*}\right)
\end{gathered}
$$

where $S_{(i)}$ denotes the screening charge constructed from $\vartheta_{i}$. It is then immediate to show that in the elliptic sector,

$$
\left(e_{(1)}^{-J \alpha_{-} \Phi}\right)^{\dagger}=e_{(2)}^{-J \alpha_{-} \Phi} \quad\left(\varpi=\varpi^{*}\right)
$$

\footnotetext{
${ }^{9}$ Actually the last factor in Eq.4.19 removes only $\mu_{0}^{m}$ in Eq.4.13, but the remaining normalization constant $\mu_{0}^{J}$ plays no role here. It will become important, however, when we consider the equations of motion.
} 
Our exponentials can formally be interpreted also in the hyperbolic sector, and fulfill there

$$
\left(e_{(i)}^{-J \alpha_{-} \Phi}\right)^{\dagger}=e_{(i)}^{-J \alpha_{-} \Phi} \quad\left(\varpi=-\varpi^{*}\right)
$$

However, their locality properties are not entirely obvious in this sector-cf. below. Returning to the elliptic case, we note that Eq.4.17 and Eq.4.23 imply

$$
\left(T e_{(1)}^{-J \alpha_{-} \Phi} T^{-1}\right)^{\dagger}=C\left(T e_{(1)}^{-J \alpha_{-} \Phi} T^{-1}\right) C^{-1}
$$

with

$$
C(\varpi, \bar{\varpi})=T^{-1 \dagger}(\varpi, \bar{\varpi}) T^{-1}(-\varpi,-\bar{\varpi})
$$

Thus, hermiticity is realized only up to a similarity transformation. In fact, in the elliptic sector there exists no similarity transformation $T$ at all such that $C$ becomes trivial, even if Eq.4.17 is not imposed. This fact was first observed later rediscovered in [30]. Nevertheless, the weaker hermiticity property Eq.4.25 serves almost the same purpose as "true" hermiticity as far as correlators of the Liouville exponentials are concerned, as the similarity transformation $C$ cancels out up to the contributions from the end points where $C$ resp. $C^{-1}$ hits the left resp. right vacuum. A more serious problem in the elliptic sector, also observed in ref. 21, is that the Liouville exponentials possess no natural restriction to the subspace of positive norm states, given by the condition $|\varpi|<1+\pi / h,|\bar{\varpi}|<1+\pi / h$. For the coupling of $c<1$ matter to gravity, however, this problem is irrelevant, as all negative norm states become decoupled through the Virasoro conditions.

\subsection{The Liouville Field $\Phi$}

\subsubsection{Definition}

Having constructed Liouville exponentials with arbitrary continous spins, we can now define the Liouville field $\Phi$ itself by 27

$$
\alpha_{-} \Phi:=-\left.\frac{d}{d J} e^{-J \alpha_{-} \Phi}\right|_{J=0}
$$

Though $\Phi$ is not really a primary field - it is similar to the stress-energy tensor in this respect - it is needed to verify the validity of canonical commutation relations and the quantum equations of motion. Thus we expand Eq4.15 near $J=0$. In this limit, the factor $\prod_{k=1}^{J+m}\lfloor 1+2 J-k\rfloor \rightarrow \frac{2 J h}{\sin h} \prod_{k=2}^{J+m}\lfloor 1+2 J-k\rfloor$ vanishes except for $J+m=0$, and the exponential tends to one as it should. It then follows immediately that

$$
\begin{gathered}
\Phi(\sigma, \tau)=-\left(\vartheta_{1}(u)+\bar{\vartheta}_{1}(v)\right)+\frac{2 h}{\alpha_{-} \sin h} \times \\
\sum_{n=1}^{\infty} \tilde{\mu}_{0}^{n} \frac{1}{\lfloor n\rfloor} \prod_{k=1}^{n} \frac{1}{\lfloor\varpi+2 n-k\rfloor\lfloor\varpi+k\rfloor} S(u)^{n} \bar{S}(v)^{n} .
\end{gathered}
$$

\footnotetext{
${ }^{10}$ More precisely, it was pointed out in the second of refs. 21 that $e^{-\alpha_{-} \Phi / 2}$ can be chosen hermitian resp. antihermitian in certain regions of $\varpi, \bar{\varpi}$ space, but $e^{-\alpha_{-} \Phi / 2}$ cannot be consistently restricted to these regions.
} 


\subsubsection{Periodicity properties and singularity structure}

In the hyperbolic sector where $\varpi=\bar{\varpi}$, the Liouville field of Eq.4.27 is manifestly periodic. However, inspecting the periodicity behaviour of $\Phi$ in the elliptic sector with $\varpi \neq \bar{\varpi}$, we find that

$$
\alpha_{-} \Phi(\sigma+2 \pi, \tau)=\alpha_{-} \Phi(\sigma, \tau)-2 \pi i k
$$

where $k$ is the parameter appearing in Eq4.1. The constant is entirely produced by the free field contribution to $\Phi$, as the series in screening charges is periodic order by order (cf. Eq.3.4). Eq.4.28 obviously calls for some explanation, as at least classically the Liouville field should be periodic by definition. We will carry out the discussion classically, but this will suffice to obtain a qualitative understanding of the situation. The essential point is that the definitions Eq.2.2 and Eq.4.26, though seemingly equivalent classically, actually differ slightly in the elliptic sector. If $2 \sqrt{\gamma} \Phi(\sigma, \tau)$ is regular everywhere in $[0,2 \pi]$, the two definitions clearly can differ only by a constant. But it is well known[22] that in the elliptic sector (with $k \neq 0$ ) there are $|k|$ nonintersecting singularity lines, thus $|k|$ singularities in $\sigma \in[0,2 \pi]$. At a singular point, the constant connecting the two definitions may - and does - change, creating in this way a nontrivial periodicity behaviour of our field $\Phi$ of Eqs.4.26, 4.27. Indeed, classically the definition Eq.4.26 is equivalent to

$$
2 \sqrt{\gamma} \Phi=-2 \ln A^{\prime-1 / 2}-2 \ln B B^{\prime-1 / 2}-2 \ln (1-A / B)+\text { const. }
$$

with the series expansion representing the logarithm. It follows easily from the results of 22] that at each singular point, $2 \ln (1-A / B)$ - and hence $\Phi$ of Eq.4.29 jumps by an imaginary constant $-2 \pi i \operatorname{sgn} k$, whereas there is no such jump, of course, in Eq.2.2 which is by definition real. Hence,

$$
\begin{gathered}
2 \sqrt{\gamma}\left(\Phi_{E q .22}(\sigma+2 \pi, \tau)-\Phi_{E q .4 .26}(\sigma, \tau)\right) \\
-2 \sqrt{\gamma}\left(\Phi_{E q .2 .2}(\sigma+2 \pi, \tau)-\Phi_{E q .2 .2(\sigma, \tau))=-2 \pi i k}\right.
\end{gathered}
$$

As the second difference is zero, we reproduce Eq.4.28. Thus the Liouville field we are using differs from the "true" one only by a constant between any two singularity lines, but the constant changes at the singularities. In particular, our $\Phi$ cannot be real everywhere in $\sigma \in[0,2 \pi]$. (This has nothing to do with the nonhermiticity of the exponentials noted in section 4.1.6, as the latter is independent of $\sigma$; indeed, for the exponentials with half-integer $J$, the jumps play no role for the hermiticity behaviour). We remark that the periodicity behaviour of our Liouville field is actually quite natural, as the spectrum in the elliptic sector contains a winding number $(k)$ and therefore looks like that of a compactified field.

When using the free field $\vartheta_{2}$ instead of $\vartheta_{1}$, the periodicity behaviour of $\Phi$ of Eq.4.27 will be exactly opposite. One may think that the regions of convergence for the two (classical) expansions, $|A / B|<1$ resp. $|B / A|<1$ are complementary and therefore there is no contradiction. However, in contrast to the hyperbolic sector we have $|A / B| \equiv 1$ in the elliptic sector, such that both series expansions are exactly 
on their circle of convergence, and in fact converge there except for the singularities at $A=B$. Thus we see that $\vartheta_{1} \leftrightarrow \vartheta_{2}$ invariance is broken by the singularities in the elliptic sector. However, for the exponentials with half-integer $J$, we get the same periodicity behaviour for the $\vartheta_{1}$ and the $\vartheta_{2}$ representation. Correspondingly, we were able even in the quantum case to construct these exponentials in a $\vartheta_{1} \leftrightarrow \vartheta_{2}$ invariant way. For continous $J$, however, it is not obvious how this invariance can be restored.

\subsubsection{The case $k=0$}

A special consideration is required for the case where $\varpi$ is real (elliptic sector) but $k=0$ in Eq.4.1. It follows from the work of [22] that in this situation, there is no real Liouville field even classically. However, from the point of view of the locality analysis, the case $k=0$ is very natural and therefore we did not exclude it. It is not hard to show that indeed more generally one needs to have

$$
\varpi \bar{\varpi}<0
$$

classically in order to obtain real solutions of the Liouville equation with positive cosmological constant. In the other case, one has $2 \sqrt{\gamma} \operatorname{Im} \Phi= \pm i \pi$, and so the real part of $\Phi$ solves the Liouville equation with negative cosmological constant. As regards the singularity structure, $\sigma$ and $\tau$ essentially exchange their roles, and thus one obtains timelike instead of spacelike singularity lines 29]. The number of singularities can in general be greater than $|k|$, though $|k|$ continues to characterize the periodicity behaviour of our solution Eq.4.27. The explanation is that the additional singularities always come in pairs with opposite associated jumps $\pm 2 \pi i$ of $2 \sqrt{\gamma} \Phi$, so their effect is not seen in the overall periodicity behaviour of $\Phi$. In particular, for $k=0$ we now understand why the Liouville field Eq.4.27 is periodic in spite of the possible presence of singularities. We stress also that in our analysis there is no restriction on the sign of $\varpi \bar{\varpi}$, thus we describe solutions of the Liouville equation with both signs of the cosmological constant.

\subsubsection{The field equations}

For the considerations in this subsection and below, the similarity transformations $T$ discussed above play no role and so we return to the representation Eq.4.27 resp. Eq.4.15 of the Liouville exponentials. It is straightforward to derive from Eq.4.27 explicit expressions for the derivatives of $\Phi$, and one obtains:

$$
\begin{gathered}
\partial_{u} \Phi=-\partial_{u} \vartheta_{1}+\frac{2 i h}{\alpha_{-} \sin h} \sum_{n=1}^{\infty} \frac{\tilde{\mu}_{0}^{n}}{\lfloor n\rfloor} c_{n}(\varpi) \times \\
\prod_{k=1}^{n} \frac{1}{\lfloor\varpi+2 n-k\rfloor\lfloor\varpi+k\rfloor} U_{n}^{(-1)} \bar{S}^{n},
\end{gathered}
$$

with

$$
c_{n}(\varpi)=2 \sin h q^{\varpi+1}\lfloor n\rfloor\lfloor\varpi+n\rfloor
$$


and similarly for $\partial_{v} \Phi$. In Eq.4.32 we observe the appearance of the coefficients of the expansion Eq.4.15 with $J=-1$. Taking the crossed derivative we thus get

$$
\partial_{u} \partial_{v} \Phi=-\frac{\alpha_{-}}{8} e^{\alpha_{-} \Phi}
$$

if we choose

$$
\tilde{\mu}_{0}=\frac{1}{32 \pi \sin h}
$$

Eq.4.34 is the quantum Liouville field equation associated with an action given by Eq.2.1 with $2 \sqrt{\gamma}$ replaced by $\alpha_{-}$, and with $\mu=1$. In view of the recently shown equivalence of different frameworks 25, we can directly compare this result with the one obtained in an older analysis by Otto and Weigt [27], and find agreement $\square$. At this point it should be noted that the main approaches within the operator framework, those of refs. 28], refs. 27], and the present one, have been shown in 25] to be equivalent, precisely by means of a similarity transformation of the form Eq.4.12.

\subsubsection{Equal-time commutation relations}

We now proceed to the canonical commutation relations. It follows trivially from Eqs.4.6, 4.26 that

$$
\left[\Phi(\sigma, \tau), \Phi\left(\sigma^{\prime}, \tau\right)\right]=0
$$

Next, by differentiating Eq.4.36 twice with respect to time and using the equations of motion, we see that also

$$
\left[\Pi(\sigma, \tau), \Pi\left(\sigma^{\prime}, \tau\right)\right]=0
$$

where $\Pi(\sigma, \tau)$ is the canonical momentum,

$$
\Pi(\sigma, \tau)=\frac{1}{4 \pi} \partial_{\tau} \Phi(\sigma, \tau)
$$

Furthermore we note that $\left[\Pi(\sigma, \tau), \Phi\left(\sigma^{\prime}, \tau\right)\right]$ can be nonvanishing only at $\sigma=\sigma^{\prime}$, due to the fact the $R$-matrices for arbitrary spins depend on $\tau, \sigma$ only via the step functions $\theta\left(u-u^{\prime}\right)$ resp. $\theta\left(v-v^{\prime}\right)$. On the other hand, the contribution of the free field parts of $\Pi$ and $\Phi$ gives precisely the expected result:

$$
\left[\Pi(\sigma, \tau), \Phi\left(\sigma^{\prime}, \tau\right)\right] \mid \text { free field }=-i \delta\left(\sigma-\sigma^{\prime}\right)
$$

By straightforward arguments [26] one can show that the sum of the other contributions vanishes. Thus one finds for the full commutator,

$$
\left[\Pi(\sigma, \tau), \Phi\left(\sigma^{\prime}, \tau\right)\right]=-i \delta\left(\sigma-\sigma^{\prime}\right)
$$

as expected, showing that the quantization scheme is indeed canonical.

\footnotetext{
11 Note that the formula of Otto and Weigt quoted in ref.25 needs to be multiplied by a factor $\left(\frac{\sin h}{h}\right)^{2 J}$ to be in accord with the equations of motion. (This was already noticed in [27]).
} 


\section{The case of two screening charges.}

\subsection{The braiding}

In the above analysis, we have used only one quantum deformation parameter $h$, which is related to the semiclassical screening charge $\alpha_{-}$according to Eq.3.5 and tends to zero in the classical limit $C \rightarrow \infty$ according to Eq.2.19. However, it is of course well known e.g. from the minimal models that there exists another screening charge $\alpha_{+}$with analogous properties, except that it blows up in the classical limit. Correspondingly, there is a second deformation parameter $\widehat{h}$ with

$$
\alpha_{+} \equiv 2 \sqrt{\frac{\widehat{h}}{2 \pi}}
$$

(cf. Eq.3.5), and $\widehat{h}$ is given by Eq.2.19 with the other sign of the square root. The relation between $h$ and $\widehat{h}$ is simply

$$
\widehat{h} \equiv \pi^{2} / h
$$

Thus we obtain another set of screening operators $\widehat{S}$ resp. primary fields $\widehat{U}_{\widehat{m}}^{(\widehat{J})}$ which have the same form Eqs.3.3, 3.6 as $S$ resp. $U_{m}^{(J)}$, but involve $\widehat{h}$ instead of $h$. Hence their exchange algebra (and also their fusion properties) are the same as that of the "unhatted" operators. More generally, one can combine the fields $\widehat{U}_{\widehat{m}}^{(\widehat{J})}$ and $U_{m}^{(J)}$ by fusion to obtain the operators $U_{m \widehat{m}}^{(J \widehat{J})}$ with the properties (cf. Eqs.3.2, 3.7)

$$
\begin{gathered}
\Delta_{J, \widehat{J}}=\Delta_{J+\widehat{J} \pi / h} \\
U_{m m}^{(J \widehat{\jmath})} \varpi=(\varpi+2 m+2 \widehat{m} \pi / h) U_{m \bar{m}}^{(J \widehat{\widehat{J}})}, U_{m \bar{m}}^{(J \widehat{\jmath})} \widehat{\varpi}=(\widehat{\varpi}+2 \widehat{m}+2 m h / \pi) U_{m m}^{(J \widehat{J})}
\end{gathered}
$$

where $\widehat{\varpi}=\varpi h / \pi$. For half-integer $J, \widehat{J}$ the braiding (and fusion) of the $U_{m \widehat{m}}^{(J \widehat{J})}$ follows immediately from that of the $U_{m}^{(J)}$ because the two sets of operators $U_{m}^{(J)}$ and $\widehat{U}_{\widehat{m}}^{(\widehat{J})}$ commute up to a phase:

$$
U_{m}^{(J)}(\sigma) \widehat{U}_{\widehat{m}}^{(\widehat{J})}\left(\sigma^{\prime}\right)=e^{-2 \pi i J \widehat{J} \epsilon\left(\sigma-\sigma^{\prime}\right)} \widehat{U}_{\widehat{m}}^{(\widehat{J})}\left(\sigma^{\prime}\right) U_{m}^{(J)}(\sigma)
$$

The natural expectation is that this will remain true even for noninteger $2 J$. However, here we meet a surprise. The commutation of $V_{-J}^{(J)}(\sigma)$ and $\widehat{V}_{-\widehat{J}}^{(J)}\left(\sigma^{\prime}\right)$ gives the factor in Eq.5.4, the screening charges $S(\sigma)$ and $\hat{S}\left(\sigma^{\prime}\right)$ commute, but

$$
S_{\sigma \sigma^{\prime}} \widehat{V}_{-\widehat{J}}^{(\widehat{J})}\left(\sigma^{\prime}\right)=e^{2 \pi i \hat{J}} \widehat{V}_{-\widehat{J}}^{(\widehat{J})}\left(\sigma^{\prime}\right) S_{\sigma \sigma^{\prime}}
$$

whereas

$$
S_{\Delta} \widehat{V}_{-\widehat{J}}^{(\widehat{J})}\left(\sigma^{\prime}\right)=e^{-2 \pi i \widehat{J}} \widehat{V}_{-\widehat{J}}^{(\widehat{J})}\left(\sigma^{\prime}\right) S_{\Delta}
$$


The phase factors agree only when $2 J$ is integer, and thus the commutation of hatted and unhatted operators becomes nontrivial in general. We should therefore restart the machinery of section 2 with the operators $U_{m \widehat{m}}^{(J \widehat{J})}$, where

$$
U_{m \widehat{m}}^{(J \widehat{J})}:=V_{-J}^{(J)} \widehat{V}_{-\widehat{J}}^{(\widehat{J})} S^{J+m} \hat{S}^{\widehat{J}+\widehat{m}}
$$

with the product of the first two factors being defined by renormalizing the shortdistance singularity as usual (cf. also ref.[12]). One obtains the following result for the $R$-matrix of the $U_{m \widehat{m}}^{(J \widehat{J})}$ :

$$
R_{U}\left(\underline{J}, \underline{J^{\prime}} ; \varpi\right) \underline{\underline{m}}_{2} \frac{\underline{m}_{1}}{\underline{m}^{\prime}}=q^{-J^{e} J^{e \prime}} \widehat{q}^{-\widehat{J}^{e} \widehat{J}^{e^{\prime}}} R_{U}\left(J^{e}, J^{e \prime} ; \varpi\right)_{m^{\circ} m^{\circ}}^{m_{0}^{\circ} m^{\circ}} \hat{R}_{U}\left(\widehat{J}^{e}, \widehat{J}^{e^{\prime}} ; \widehat{\varpi}\right)_{\widehat{m}^{\circ} \widehat{m}^{\circ}}^{{\widehat{m^{\circ}}}_{\widehat{m}^{\circ}}}
$$

Here, we have introduced "effective spins"

$$
J^{e}:=J+\widehat{J} \pi / h \quad, \quad \widehat{J}^{e}:=\widehat{J}+J h / \pi
$$

and shifted $m$ 's

$$
\begin{gathered}
m^{\circ}=m-\widehat{J} \frac{\pi}{h}, \quad m^{\circ \prime}=m^{\prime}-\widehat{J}^{\prime} \frac{\pi}{h}, \quad m_{1}^{\circ}=m_{1}-\widehat{J} \frac{\pi}{h}, \quad m_{2}^{\circ}=m_{2}-\widehat{J}^{\prime} \frac{\pi}{h} \\
\widehat{m}^{\circ}=\widehat{m}-J \frac{h}{\pi}, \quad \widehat{m}^{\circ \prime}=\widehat{m}-J^{\prime} \frac{h}{\pi}, \quad \widehat{m}_{1}^{\circ}=\widehat{m}_{1}-J \frac{h}{\pi}, \quad \widehat{m}_{2}^{\circ}=\widehat{m}_{2}-J^{\prime} \frac{h}{\pi} .
\end{gathered}
$$

Their appearance is easy to understand upon noting that $V_{-J}^{(J)} \widehat{V}_{-\widehat{J}}^{(\widehat{J})} \propto V_{-J^{e}}^{\left(J^{e}\right)}$. So indeed everything can depend only on the effective spins and the screening numbers

$$
\begin{aligned}
& n=J+m=J^{e}+m^{\circ}, \quad n^{\prime}=J^{\prime}+m^{\prime}=J^{e \prime}+m^{\circ \prime}, \\
& \widehat{n}=\widehat{J}+\widehat{m}=\widehat{J}^{e}+\widehat{m}^{\circ}, \quad \widehat{n}^{\prime}=\widehat{J}^{\prime}+\widehat{m}^{\prime}=\widehat{J}^{e}+\widehat{m}^{\circ \prime}
\end{aligned}
$$

and similarly for $n_{1}, n_{2}, \widehat{n}_{1}, \widehat{n}_{2}$ (with $n+n^{\prime}=n_{1}+n_{2}, \widehat{n}+\widehat{n}^{\prime}=\widehat{n}_{1}+\widehat{n}_{2}$ ). The $R$-matrices $R_{U}$ and $\hat{R}_{U}$ in Eq.5.8 are given by the same expression Eq.3.21, written in terms of the deformation parameter $h$ resp. $\widehat{h}$.

Thus in the continuous case $2 J$ and $2 \widehat{J}$ loose meaning, since it is not possible to recover them from $J^{e}$. Correspondingly, the fields $U_{m \widehat{m}}^{(J \widehat{J})}$ should be thought of as related to a new quantum group $U_{q \hat{q}}$ whose representations are labelled by the effective spin $J^{e}$ and carry two magnetic quantum numbers $m^{\circ}, \widehat{m}^{\circ}$ instead of one. A more detailed elaboration of its properties is in preparation. We can write the complete $R$-matrix again in the form Eq.3.21, if we introduce "vectorial" quantities

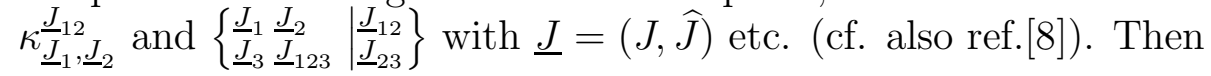

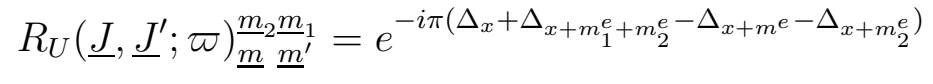

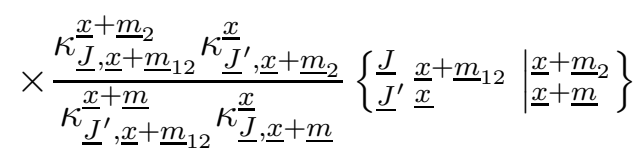


where

$$
\kappa_{\underline{J}, \underline{x}+\underline{m}}^{x}=\kappa_{J^{e}, x+m^{\circ}}^{x} \widehat{\kappa}_{\widehat{J}^{e}, \widehat{x}+\widehat{m}^{\circ}}
$$

and

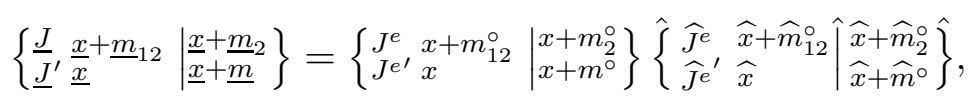

where we have let $m_{12}^{\circ}=m_{1}^{\circ}+m_{2}^{\circ}, \widehat{m}_{12}^{\circ}=\widehat{m}_{1}^{\circ}+\widehat{m}_{2}^{\circ}$ and $m^{e}=m+\widehat{m} \pi / h$. We note that Eq.5.12 agrees precisely with the "vectorial" $6 j$ symbol found in ref.[17] where extensions of the usual $6 j$ are discussed on the basis of the polynomial equations. This completes the derivation of the braiding of the chiral vertex operators with both screening charges. We add as a final remark that the generalized $6 j$ symbols fulfill orthogonality relations completely analogous to those known for the usual ones. They take the form

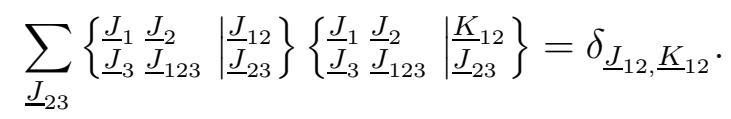

where the summation is over all $J^{e}{ }_{23}$ such that $J_{2}^{e}+J^{e}{ }_{3}-J^{e}{ }_{23} \in \mathbf{Z}_{+}+\pi / h \mathbf{Z}_{+}$.

\subsection{The generalized Liouville field}

The generalization of the Liouville exponential is given by

$$
e^{-J^{e} \alpha_{-} \underline{\Phi}(\sigma, \tau)}=\sum_{n, \widehat{n}=0}^{\infty} \mu_{0}^{n} \widehat{\mu}_{0}^{n} \widetilde{V}_{n \widehat{n}}^{\left(J^{e}\right)}(u) \overline{\widetilde{V}}_{n \widehat{n}}^{\left(J^{e}\right)}(v)
$$

where $\widehat{\mu}_{0}$ is given by equations similar to Eqs.4.16 and 4.35 with $h \rightarrow \widehat{h}$. Of course, the preference of $\alpha_{-}$over $\alpha_{+}$in Eq.5.14 is purely notational as $e^{-J^{e} \alpha_{-}} \underline{\Phi} \equiv e^{-\widehat{J}^{e} \alpha_{+}} \underline{\Phi}$. Using Eq.5.13, it is straightforward to verify that the generalized exponential is local and closed by fusion, provided $\varpi=\bar{\varpi}$. The previous discussions of the hermiticity and Weyl reflection properties can be easily generalized. We now turn to the generalized Liouville field, which we define again by Eq.4.26. Thus we obtain

$$
\begin{gathered}
\underline{\Phi}(\sigma, \tau)=-\left(\vartheta_{1}(u)+\bar{\vartheta}_{1}(v)\right) \\
+\frac{2 h}{\alpha_{-} \sin h} \sum_{n=1}^{\infty} \tilde{\mu}_{0}^{n} \frac{1}{\lfloor n\rfloor} \prod_{k=1}^{n} \frac{1}{\lfloor\varpi+2 n-k\rfloor\lfloor\varpi+k\rfloor} S(u)^{n} \bar{S}(v)^{n} . \\
+\frac{2 \widehat{h}}{\alpha_{+} \sin \hat{h}} \sum_{\widehat{n}=1}^{\infty} \tilde{\widehat{\mu}}_{0}^{n} \frac{1}{\hat{\lfloor} \hat{n} \hat{\rfloor}} \prod_{\hat{k}=1}^{\widehat{n}} \frac{1}{\hat{\lfloor} \widehat{\varpi}+2 \widehat{n}-\hat{k} \hat{\jmath} \hat{\lfloor} \widehat{\varpi}+\hat{k} \hat{\rfloor}} \widehat{S}(u)^{\widehat{n}} \widehat{\bar{S}}(v)^{\hat{n}} .
\end{gathered}
$$

It differs from the previous one by the last line. Since $\varpi=\bar{\varpi}, \Phi$ is periodic. As a result, the quantum field equation becomes

$$
\partial_{u} \partial_{v} \underline{\Phi}=-\frac{\alpha_{-}}{8} e^{\alpha_{-} \Phi}-\frac{\alpha_{+}}{8} e^{\alpha_{+} \hat{\Phi}}
$$

involving both cosmological terms . Since $\underline{\Phi}=\Phi+\hat{\Phi}+\vartheta_{1}+\bar{\vartheta}_{1}$, the validity of Eq.5.16 is a trivial consequence of the equations of motion with a single screening 
charge. The reason why $\underline{\Phi}$ must be shifted w.r.t. $\Phi+\hat{\Phi}$ is that $\Phi+\hat{\Phi}$ alone is not local. Indeed, using Eqs.4.14 and its left-moving analog, and the fact that screening charges of different type commute, we see that the only nonzero contributions to $\left[\Phi(\sigma, \tau)+\hat{\Phi}(\sigma, \tau), \Phi\left(\sigma^{\prime}, \tau\right)+\hat{\Phi}\left(\sigma^{\prime}, \tau\right)\right]=\left[\Phi(\sigma, \tau), \hat{\Phi}\left(\sigma^{\prime}, \tau\right)\right]+\left[\hat{\Phi}(\sigma, \tau), \Phi\left(\sigma^{\prime}, \tau\right)\right]$ are of the form $\left[\vartheta_{1}, \widehat{S}\right]$ resp. $\left[\vartheta_{1}, S\right]$. These commutators are precisely cancelled by the free field shift. Finally, one may easily extend the previous discussion of the canonical commutation relations. The result is that Eqs.4.36, 4.37 and 4.40 remain true for the generalized Liouville field without any modification. If we regard the passage from $h$ to $\hat{h}$, or $\alpha_{-}$to $\alpha_{+}$, as some kind of duality transformation, then Eq.5.16 describes the selfdual version of the theory. It would be interesting to find a Lagrangian description of this theory -which is of course not simply given by the usual Liouville Lagrangian with both cosmological terms, as should be clear from the remarks above.

\section{Group-theoretic approach to the classical Liou- ville exponentials}

We now switch subjects completely and turn to the question of the quantum $\tau$ function. As a preparation of the coming section, it is pedagogical to temporarily return to the classical case. We will consider only the case of half-integer positive spins explicitly. Then we have the general solution of ref. [34]:

$$
\begin{gathered}
e^{-j \Phi(z, \bar{z})}=\frac{<j, j\left|\bar{M}^{-1}(\bar{z}) M(z)\right| j, j>}{(s(z) \bar{s}(\bar{z}))^{j}} \\
\frac{d M}{d z}=s(z) M j_{-}, \quad \frac{d \bar{M}}{d \bar{z}}=\bar{s}(\bar{z}) \bar{M} j_{+},
\end{gathered}
$$

where $s$ and $\bar{s}$ are arbitrary functions of a single variable. The symbols $j_{ \pm}$represent $\operatorname{sl}(2)$ generators satisfying $\left[j_{+}, j_{-}\right]=2 j_{3}$, and $\mid j, j>$ are highest-weight states $j_{+} \mid j, j>=0$. In Eq.6.1 and below, we use Euclidean coordinates on the sphere, $z=e^{\tau+i \sigma}, \bar{z}=e^{\tau-i \sigma}$. To establish the connection with the approach of the preceding sections, note first that $s, \bar{s}$ are related to the arbitrary functions $A, B$ appearing in the general classical solution Eq.2.2 by

$$
s(z)=A^{\prime}(z), \quad \bar{s}(\bar{z})=-B^{\prime}(\bar{z}) / B^{2}
$$

and thus $s$ and $\bar{s}$ can be identified with the classical equivalents of the screening charge densities $V_{1}^{(-1)}, \bar{V}_{1}^{(-1)}$ (up to normalization). It is then immediate to verify that Eq.6.1 reduces to Eq.2.5 after evaluation of the matrix element. Actually, the $S L(2, \mathbf{C})$ symmetry Eq.2.3 allows us just as well to identify $s^{-1 / 2}, \bar{s}^{-1 / 2}$ with any linear combination of the quasiperiodic (Bloch wave) fields $V_{-1 / 2}^{(1 / 2)}$ and $V_{+1 / 2}^{(1 / 2)}$ resp. $\bar{V}_{-1 / 2}^{(1 / 2)}$ and $\bar{V}_{+1 / 2}^{(1 / 2)}$. For the classical considerations below, the assignment chosen

\footnotetext{
${ }^{12}$ In this section, we redefine the Liouville field, for simplicity, so that the coupling constant need not be written any more.
} 
is irrelevant; however, on the quantum level the situation will be quite different. Looking at Eq.6.1, one may wonder why the Liouville exponential should be given by highest-weight matrix elements. The basic reason is that Eq.6.1 must be such that

$$
e^{-j_{1} \Phi(z, \bar{z})} e^{-j_{2} \Phi(z, \bar{z})}=e^{-\left(j_{1}+j_{2}\right) \Phi(z, \bar{z})},
$$

by the very definition of the classical exponential function. In order to verify this, we introduce the rescaled exponentials

$$
E^{(j)}\left(s, j_{-}\right)=s^{-j}(z) M(z)=s^{-j}(z) e^{\int^{z} s\left(z^{\prime}\right) d z^{\prime}} j_{-}
$$

resp.

$$
\bar{E}^{(j)}\left(\bar{s}, j_{+}\right)=\bar{s}^{-j}(\bar{z}) \bar{M}^{-1}(\bar{z})=\bar{s}^{-j}(\bar{z}) e^{-\int^{\bar{z}} \bar{s}\left(\bar{z}^{\prime}\right) d \bar{z}^{\prime} j_{+}}
$$

Thus the left-hand side of Eq.6.3 can be written as

$$
\begin{gathered}
e^{-j_{1} \Phi(z, \bar{z})} e^{-j_{2} \Phi(z, \bar{z})}=\left(<j_{1}, j_{1}\left|<j_{2}, j_{2}\right|\right) \bar{E}^{\left(j_{1}\right)}\left(\bar{s}, j_{+}\right) \otimes \bar{E}^{\left(j_{2}\right)}\left(\bar{s}, j_{+}\right) \times \\
E^{\left(j_{1}\right)}\left(s, j_{-}\right) \otimes E^{\left(j_{2}\right)}\left(s, j_{-}\right)\left(\left|j_{1}, j_{1}>\right| j_{2}, j_{2}>\right) \\
=\left(<j_{1}, j_{1}\left|<j_{2}, j_{2}\right|\right) \bar{E}^{\left(j_{1}+j_{2}\right)}\left(\bar{s}, j_{+} \otimes 1+1 \otimes j_{+}\right) \times \\
E^{\left(j_{1}+j_{2}\right)}\left(s, j_{-} \otimes 1+1 \otimes j_{-}\right)\left(\left|j_{1}, j_{1}>\right| j_{2}, j_{2}>\right)
\end{gathered}
$$

The highest weight-states considered are the only ones such that the tensor product gives a single irreducible representation. Its spin is $j_{1}+j_{2}$, and $\left|j_{1}, j_{1}>\right| j_{2}, j_{2}>$ is the highest-weight vector. Since the matrix element of $\bar{M}^{-1} M$ is determined solely by the group structure, it only depends upon the spin of the representation, and not upon the way it is realized; hence Eq.6.3 follows. In particular, we have

$$
e^{-j \Phi(z, \bar{z})}=\left(e^{-(1 / 2) \Phi(z, \bar{z})}\right)^{2 j}
$$

and thus Eq.6.1 may be re-written using binomial coefficients (more on this below).

It is well-known (see e.g. ref.[16]) that the Liouville solution and its Toda generalization are actually tau-functions in the sense of the Kyoto group 23. A characteristic feature of tau-functions is to involve highest-weight states. We shall not dwell into the precise connection, since it is not directly evident from Eq.6.1. We shall rather recall the existence of bilinear equations of the Hirota type, which was the original motivation to introduce tau functions. The method of derivation we will use is not the same as the standard ones of ref. 223] or ref. [24]. Its interest is that it will remain applicable in the quantum case.

One may obtain a closed equation for the $j=1 / 2$ Liouville exponential as follows. Making use of Eq.6.1, let us compute the antisymmetric bilinear expression

$$
e^{-\Phi / 2} \partial_{z} \partial_{\bar{z}} e^{-\Phi / 2}-\partial_{z} e^{-\Phi / 2} \partial_{\bar{z}} e^{-\Phi / 2}=
$$

\footnotetext{
${ }^{13}$ For the conceptual considerations here, the precise normalization of the $E^{(j)}$ is not important. Therefore we don't specify the lower integration limits in Eq.6.4.
} 


$$
\begin{aligned}
& -<\frac{1}{2}, \frac{1}{2}\left|\bar{M}^{-1} M\right| \frac{1}{2}, \frac{1}{2}><\frac{1}{2},-\frac{1}{2}\left|\bar{M}^{-1} M\right| \frac{1}{2},-\frac{1}{2}> \\
& +<\frac{1}{2},-\frac{1}{2}\left|\bar{M}^{-1} M\right| \frac{1}{2}, \frac{1}{2}><\frac{1}{2}, \frac{1}{2}\left|\bar{M}^{-1} M\right| \frac{1}{2},-\frac{1}{2}>
\end{aligned}
$$

The point of this particular combination of derivatives is that the functions $s$ and $\bar{s}$ disappear, and the result is given by the matrix element of $\overline{\mathcal{M}}^{-1} \mathcal{M}$ in the $j=0$ representation, where $\mathcal{M}=\overline{\mathcal{M}}=1$. Thus we get

$$
e^{-\Phi / 2} \partial_{z} \partial_{\bar{z}} e^{-\Phi / 2}-\partial_{z} e^{-\Phi / 2} \partial_{\bar{z}} e^{-\Phi / 2}=-1
$$

Of course it is trivial to rederive this equation directly from the Liouville equation; however this form - the simplest example of Hirota bilinear equations - which only makes use of the Liouville exponentials and not of the field $\Phi$ itself, will be much easier to generalise to the quantum case. For later use we note that this Hirota equation is equivalent to the following relation in the Taylor expansion for $z^{\prime} \rightarrow z$, $\bar{z}^{\prime} \rightarrow \bar{z}$.

$$
e^{-\Phi / 2\left(z^{\prime}, \bar{z}^{\prime}\right)} e^{-\Phi / 2(z, \bar{z})}-e^{-\Phi / 2\left(z^{\prime}, \bar{z}\right)} e^{-\Phi / 2\left(z, \bar{z}^{\prime}\right)} \sim-\left(z^{\prime}-z\right)\left(\bar{z}^{\prime}-\bar{z}\right)
$$

Clearly we may obtain other bilinear equations for $\exp (-j \Phi)$ with $j \neq 1 / 2$ by again projecting out the $j=0$ component of the product.

\section{Liouville exponentials and q-deformations}

\subsection{The quantum group structure}

The deep connection of the quantum Liouville theory with $U_{q}(\operatorname{sl}(2))$ was there from the beginning[11], but in disguise. It was elucidated more recently in refs.[目, 2, 田, 8, 9]. Though the fusing and braiding matrices of the $V_{m}^{(J)}$ fields are given in terms of quantum group $(6 j)$ symbols, they are not quantum group covariant; there exists another basis of chiral vertex operators $\xi_{M}^{(J)}$, related to the $V_{m}^{(J)}$ by a linear transformation, which behave as spin $J$ representations of $U_{q}(\operatorname{sl}(2))$. The transformation takes the form [2] [6] [9]

$$
\left.\xi_{M}^{(J)}(\sigma)=\mid J, \varpi\right)_{M}^{m} E_{m}^{(J)}(\varpi) V_{m}^{(J)}
$$

where $\mid J, \varpi)_{M}^{m}$ and $E_{m}^{(J)}(\varpi)$ are suitable transformation coefficients, whose explicit values are not important for our purposes (they can be found in refs. [2] [6] [9], for the case of half-integer positive spins which we consider here). Their fusing and braiding matrices are given by q Clebsch-Gordan and universal R-matrix elements respectively. In particular, the operator-product algebra of the $\xi_{M}^{(J)}$ corresponds to making q tensor products of representations. We anticipate therefore that it is this basis which should be used when trying to extend the considerations of the previous section to the quantum level, so that the arbitrariness mentioned below Eq.6.2 in 
the precise assignment of $s(z), \bar{s}(\bar{z})$ is lifted. It was shown in ref. $\| \overline{7}$ that the Liouville exponentials take the form $(2 J$ positive integer)

$$
e^{-J \alpha_{-} \Phi(z, \bar{z})}=\sum_{M=-J}^{J}(-1)^{J+M} e^{i h(J+M)} \xi_{M}^{(J)}(z) \bar{\xi}_{-M}^{(J)}(\bar{z})
$$

The point of this section is to show that with this last expression for the Liouville exponential, its quantum properties are directly connected with their classical analogues by standard q deformations.

\subsection{Connection with q-binomials}

We shall use the notations of refs. [2], [4]- 9 . One introduces

$$
\lfloor x\rfloor=\frac{\sin (h x)}{\sin (h)} .
$$

The q-deformed binomial coefficients noted $\left(\begin{array}{l}P \\ Q\end{array}\right)$ are defined by

$$
\left(\begin{array}{l}
P \\
Q
\end{array}\right):=\frac{\lfloor P\rfloor !}{\lfloor Q\rfloor !\lfloor P-Q\rfloor !}, \quad\lfloor n\rfloor !:=\prod_{r=1}^{n}\lfloor r\rfloor,
$$

They are binomial coefficients for the expansion of $(x+y)^{2 J}$, with $x$ and $y$ noncommuting variables such that

$$
x y=y x e^{-2 i h} .
$$

Indeed, it is easy to verify that they satisfy

$$
\left(\begin{array}{c}
m+1 \\
n
\end{array}\right)=e^{i h n}\left(\begin{array}{c}
m \\
n
\end{array}\right)+e^{-i h(m-n+1)}\left(\begin{array}{c}
m \\
n-1
\end{array}\right) .
$$

As a result one sees that if one lets

$$
(x+y)^{N}=\sum_{r=0}^{N}\left(\begin{array}{c}
N \\
r
\end{array}\right) e^{i h r(N-r)} x^{r} y^{N-r}
$$

one has, as required,

$$
(x+y)^{N+1}=(x+y)(x+y)^{N} .
$$

We now use the leading-order fusion properties of the $\xi$ fields [2]: to leading order in the short distance singularity at $z \rightarrow z^{\prime}$, the product of $\xi$ fields behaves as

$$
\begin{gathered}
\xi_{M}^{(J)}(z) \xi_{M^{\prime}}^{\left(J^{\prime}\right)}\left(z^{\prime}\right) \sim\left(z-z^{\prime}\right)^{-2 J J^{\prime} h / \pi} \lambda\left(J, M ; J^{\prime}, M^{\prime}\right) \xi_{M+M^{\prime}}^{\left(J+J^{\prime}\right)}\left(z^{\prime}\right), \\
\lambda\left(J, M ; J^{\prime}, M^{\prime}\right)=\sqrt{\frac{\left(\begin{array}{c}
2 J \\
J+M
\end{array}\right)\left(\begin{array}{c}
2 J^{\prime} \\
J^{\prime}+M^{\prime}
\end{array}\right)}{2 J+2 J^{\prime}}} e^{i h\left(M^{\prime} J-M J^{\prime}\right)} .
\end{gathered}
$$

\footnotetext{
${ }^{14} \mathrm{We}$ are working here on the sphere, rather than on the cylinder.
} 
Thus, if we redefine

$$
\eta_{M}^{(J)} \equiv \xi_{M}^{(J)} / \sqrt{\left(\begin{array}{c}
2 J \\
J+M
\end{array}\right)}
$$

we have

$$
\eta_{M}^{(J)} \eta_{M^{\prime}}^{\left(J^{\prime}\right)} \sim \eta_{M+M^{\prime}}^{\left(J+J^{\prime}\right)} e^{i h\left(M^{\prime} J-M J^{\prime}\right)}
$$

and thus

$$
\eta_{M}^{(J)} \sim\left(\eta_{1 / 2}^{(1 / 2)}\right)^{J+M}\left(\eta_{-1 / 2}^{(1 / 2)}\right)^{J-M} e^{\frac{i h}{2}\left(J^{2}-M^{2}\right)}
$$

In the above formulae and hereafter the symbol $\sim$ means leading term of the shortdistance expansion, divided by the singular short distance factor appearing in Eq.7.9. In terms of the $\eta$ fields, the Liouville exponential takes the form

$$
e^{-J \alpha_{-} \Phi(z, \bar{z})}=\sum_{J+M=0}^{2 J}\left(\begin{array}{c}
2 J \\
J+M
\end{array}\right)(-1)^{J+M} e^{i h(J+M)} \eta_{M}^{(J)}(z) \bar{\eta}_{-M}^{(J)}(\bar{z})
$$

or, using Eq.7.12,

$$
\begin{gathered}
e^{-J \alpha_{-} \Phi(z, \bar{z})} \sim \sum_{J+M=0}^{2 J}\left(\begin{array}{c}
2 J \\
J+M
\end{array}\right) e^{i h(J+M)(J-M)} \times \\
\left(-e^{i h} \eta_{1 / 2}^{(1 / 2)} \bar{\eta}_{-1 / 2}^{(1 / 2)}\right)^{J+M}\left(\eta_{-1 / 2}^{(1 / 2)} \bar{\eta}_{1 / 2}^{(1 / 2)}\right)^{J-M}
\end{gathered}
$$

which is completely analogous to the q-binomial expansion Eq.7.7, if we identify $x=-e^{i h} \eta_{1 / 2}^{(1 / 2)}(z) \bar{\eta}_{-1 / 2}^{(1 / 2)}(\bar{z}), \quad y=\eta_{-1 / 2}^{(1 / 2)}(z) \bar{\eta}_{1 / 2}^{(1 / 2)}(\bar{z})$. To avoid any possible confusion, we remark that Eq.7.5 does not imply that the fields $\eta_{1 / 2}^{(1 / 2)}(z) \bar{\eta}_{-1 / 2}^{(1 / 2)}(\bar{z})$ and $\eta_{-1 / 2}^{(1 / 2)}\left(z^{\prime}\right) \bar{\eta}_{1 / 2}^{(1 / 2)}\left(\bar{z}^{\prime}\right)$ commute up to a factor; this is true only in the limit $z^{\prime} \rightarrow z, \bar{z}^{\prime} \rightarrow \bar{z}$. We remark that, in view of the above construction of arbitrary Liouville exponentials, Eq.7.13 can easily be generalized to continous spins; however, this is not necessary for the points we want to make here.

\subsection{Liouville exponentials as quantum tau-functions}

As is well known, the binomial coefficients are closely related to representations of

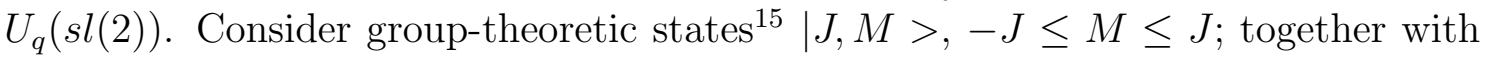
operators $J_{ \pm}, J_{3}$ such that:

$$
J_{ \pm}|J, M>=\sqrt{\lfloor J \mp M\rfloor\lfloor \pm M+1\rfloor}| J, M \pm 1>\quad J_{3}|J, M>=M| J, M>.
$$

These operators satisfy the $U_{q}(s l(2))$ commutation relations

$$
\left[J_{+}, J_{-}\right]=\left\lfloor 2 J_{3}\right\rfloor, \quad\left[J_{3}, J_{ \pm}\right]= \pm J_{ \pm} .
$$

\footnotetext{
${ }^{15}$ We assume for simplicity that $h / \pi$ is not rational.
} 
It is elementary to derive the formulae

$$
\begin{aligned}
& <J, N\left|\left(J_{+}\right)^{P}\right| J, M>=\sqrt{\frac{\lfloor J+N\rfloor !\lfloor J-M\rfloor !}{\lfloor J-N\rfloor !\lfloor J+M\rfloor !}} \delta_{N, M+P} \\
& <J, N\left|\left(J_{-}\right)^{P}\right| J, M>=\sqrt{\frac{\lfloor J-N\rfloor !\lfloor J+M\rfloor !}{\lfloor J+N\rfloor !\lfloor J-M\rfloor !}} \delta_{N, M-P}
\end{aligned}
$$

Recall further that the co-products of representations are defined by

$$
\Lambda(J)_{ \pm}=J_{ \pm} \otimes e^{i h J_{3}}+e^{-i h J_{3}} \otimes J_{ \pm}, \quad \Lambda(J)_{3}=J_{3} \otimes 1+1 \otimes J_{3}
$$

We will show now that the group-theoretical classical formulae of section 4 possess direct quantum equivalents, obtained by replacing $s l(2)$ by $U_{q}(s l(2))$. We start from the general classical solution and consider Eq.6.1 as the classical tau function. The quantum tau function should then be given by a representation of type Eq.6.1 of the operator $e^{-J \alpha_{-} \Phi}$; that is, the $q$ tau function should actually be an operator instead of a function. Let us introduce the (rescaled) q-exponentials

$$
\begin{gathered}
E_{q}^{(J)}\left(\eta(z), J_{-}\right)=\sum_{J+M=0}^{\infty} \eta_{M}^{(J)} \frac{\left(J_{-}\right)^{J+M}}{\lfloor J+M\rfloor !} \\
\bar{E}_{q}^{(J)}\left(\bar{\eta}(\bar{z}), J_{+}\right)=\sum_{J+M=0}^{\infty} e^{i h(J+M)}(-1)^{J+M} \bar{\eta}_{-M}^{(J)} \frac{\left(J_{+}\right)^{J+M}}{\lfloor J+M\rfloor !}
\end{gathered}
$$

which we take to be the quantum equivalents of the classical (rescaled) exponentials $E^{(j)}\left(s, j_{-}\right), \bar{E}^{(j)}\left(\bar{s}, j_{+}\right)$. Indeed, in the limit $h \rightarrow 0$, we see that $E_{q}^{(J)}\left(\eta, J_{-}\right)$, $\bar{E}_{q}^{(J)}\left(\bar{\eta}, J_{+}\right)$reduce to $E^{(j)}\left(s, j_{-}\right), \bar{E}^{(j)}\left(\bar{s}, j_{+}\right)$if we identify classically

$$
\begin{gathered}
\eta_{M}^{(J)}=s^{-J}(z)\left(\int^{z} s\left(z^{\prime}\right) d z^{\prime}\right)^{J+M}=\eta_{-J}^{(J)}\left(\eta_{1}^{(0)}\right)^{J+M}=\eta_{-J}^{(J)} \eta_{J+M}^{(0)} \\
\bar{\eta}_{-M}^{(J)}=\bar{s}^{-J}(\bar{z})\left(\int^{\bar{z}} \bar{s}\left(\bar{z}^{\prime}\right) d \bar{z}^{\prime}\right)^{J+M}=\bar{\eta}_{J}^{(J)}\left(\bar{\eta}_{-1}^{(0)}\right)^{J+M}=\bar{\eta}_{J}^{(J)} \bar{\eta}_{-J-M}^{(0)},
\end{gathered}
$$

which corresponds to the assignment

$$
s(z)=\eta_{1}^{(-1)}(z) \quad \bar{s}(\bar{z})=\bar{\eta}_{-1}^{(-1)}(\bar{z})
$$

Furthermore we will show now, following closely the calculation used to derive Eq.6.3 group-theoretically, that indeed the $E_{q}^{(J)}$ obey a composition law appropriate for qdeformed exponentials. The argument was inspired by ref. 38 (with an important difference- see below). In ref.[9], the fusion algebra of the $\xi$ fields was determined using the general scheme of Moore and Seiberg. One has

$$
\xi_{M_{1}}^{\left(J_{1}\right)}\left(z_{1}\right) \xi_{M_{2}}^{\left(J_{2}\right)}\left(z_{2}\right)=\sum_{J_{12}=\left|J_{1}-J_{2}\right|}^{J_{1}+J_{2}} g_{J_{1} J_{2}}^{J_{12}}\left(J_{1}, M_{1} ; J_{2}, M_{2} \mid J_{12}\right) \times
$$




$$
\sum_{\{\nu\}} \xi_{M_{1}+M_{2}}^{\left(J_{12},\{\nu\}\right)}\left(z_{2}\right)<<\varpi_{J_{12}},\{\nu\}\left|V_{J_{2}-J_{12}}^{\left(J_{1}\right)}\left(z_{1}-z_{2}\right)\right| \varpi_{J_{2}}>>
$$

where $\{\nu\}$ is a multi-index that labels the descendants, and $\mid \varpi_{J},\{\nu\}>>$ denotes the corresponding state in the Virasoro Verma-module. Similar equations hold for the $\bar{\xi}$ fields. The explicit expression of the coupling constant $g$ is not needed in the present argument. The symbol $\left(J_{1}, M_{1} ; J_{2}, M_{2} \mid J_{12}\right)$ denotes the q-Clebsch-Gordan coefficients. It follows from their very definition (see, e.g. ref.[6]) that

$$
\begin{gathered}
\sum_{M_{1}+M_{2}=M_{12}}\left(J_{1}, M_{1} ; J_{2}, M_{2} \mid J_{12}\right)\left|J_{1}, M_{1}>\otimes\right| J_{2}, M_{2}>= \\
=\frac{\left(\Lambda(J)_{-}\right)^{J_{12}-M_{12}}}{\left\lfloor J_{12}-M_{12}\right\rfloor ! \sqrt{\left(\begin{array}{c}
2 J_{12} \\
J_{12}-M_{12}
\end{array}\right)} \mid J_{12}, J_{12}>}
\end{gathered}
$$

and one finds

$$
\begin{gathered}
E_{q}^{\left(J_{1}\right)}\left(\eta\left(z_{1}\right), J_{-} \otimes 1\right) E_{q}^{\left(J_{2}\right)}\left(\eta\left(z_{2}\right), 1 \otimes J_{-}\right)\left(\left|J_{1}, J_{1}>\right| J_{2}, J_{2}>\right)= \\
\sum_{J_{12}=\left|J_{1}-J_{2}\right|}^{J_{1}+J_{2}} g_{J_{1} J_{2}}^{J_{12}} \times \\
\sum_{\{\nu\}} E_{q}^{\left(J_{12},\{\nu\}\right)}\left(\eta\left(z_{2}\right), \Lambda(J)_{-}\right)\left|J_{12}, J_{12}><<\varpi_{J_{12}},\{\nu\}\right| V_{J_{2}-J_{12}}^{\left(J_{1}\right)}\left(z_{1}-z_{2}\right) \mid \varpi_{J_{2}}>>
\end{gathered}
$$

In particular, to leading order one has

$$
\begin{gathered}
E_{q}^{\left(J_{1}\right)}\left(\eta\left(z_{1}\right), J_{-} \otimes 1\right) E_{q}^{\left(J_{2}\right)}\left(\eta\left(z_{2}\right), 1 \otimes J_{-}\right)\left(\left|J_{1}, J_{1}>\right| J_{2}, J_{2}>\right) \sim \\
E_{q}^{\left(J_{1}+J_{2}\right)}\left(\eta\left(z_{2}\right), \Lambda(J)_{-}\right) \mid J_{1}+J_{2}, J_{1}+J_{2}>.
\end{gathered}
$$

which is the natural multiplication law for q-exponentials involving quantum group generators. The coproduct is non-symmetric between the two representations. On the left hand side this comes from the non-commutativity of the $\eta$ fields as quantum field operators. Thus the present definition of q exponentials is conceptually different from the usual one where the group "parameters" are c numbers. Let us recall the latter for completeness. If on defines

$$
e_{q}(X) \equiv \sum_{r=0}^{\infty} \frac{X^{r}}{\lfloor r\rfloor !} e^{-i h r(r+1) / 2},
$$

one has

$$
e_{q}\left(x J_{ \pm} \otimes e^{i h J_{3}}\right) e_{q}\left(x e^{-i h J_{3}} \otimes J_{ \pm}\right)=e_{q}\left(x \Lambda(J)_{ \pm}\right) .
$$

Since they transform the q sum of infinitesimal generators into products, the $q$ exponentials are the natural way to exponentiate q Lie algebras. The last equation should be compared with Eq.7.25. Now the non-symmetry of the co-product is taken care of by exponentiating non-commuting group elements $\left(J_{ \pm} \otimes e^{i h J_{3}}\right.$, and $e^{-i h J_{3}} \otimes J_{ \pm}$ 
on the left-hand side), with $\mathrm{x}$ a number. This is in contrast with Eq.7.25, where $J_{ \pm} \otimes 1$, and $1 \otimes J_{ \pm}$are used instead.

Finally, we can rewrite Eq.7.13 under the form

$$
e^{-J \alpha_{-} \Phi(z, \bar{z})}=<J, J\left|\bar{E}_{q}^{(J)}\left(\bar{\eta}(\bar{z}), J_{+}\right) E_{q}^{(J)}\left(\eta(z), J_{-}\right)\right| J, J>=\tau_{q}(\eta, \bar{\eta}) .
$$

Indeed we will see that it possesses the obvious q-analogues of the properties Eq.6.5 and Eq.6.6. Hence, Eq.7.28 should be viewed as the quantum version of the LeznovSaveliev formula Eq.6.1. Since $\eta$ and $\bar{\eta}$ commute, we deduce that

$$
\begin{gathered}
e^{-J_{1} \alpha_{-} \Phi\left(z_{1}, \bar{z}_{1}\right)} e^{-J_{2} \alpha_{-} \Phi\left(z_{2}, \bar{z}_{2}\right)}= \\
\left(<J_{1}, J_{1}\left|<J_{2}, J_{2}\right|\right) \bar{E}_{q}^{\left(J_{1}\right)}\left(\bar{\eta}\left(\bar{z}_{1}\right), J_{+} \otimes 1\right) \bar{E}_{q}^{\left(J_{2}\right)}\left(\bar{\eta}\left(\bar{z}_{2}\right), 1 \otimes J_{+}\right) \\
E_{q}^{\left(J_{1}\right)}\left(\eta\left(z_{1}\right), J_{-} \otimes 1\right) E_{q}^{\left(J_{2}\right)}\left(\eta\left(z_{2}\right), 1 \otimes J_{-}\right)\left(\left|J_{1}, J_{1}>\right| J_{2}, J_{2}>\right) .
\end{gathered}
$$

Making use of the fusion algebra Eq.7.22, together with its counterpart for the $\bar{\eta}$ fields gives back the fusion algebra of the Liouville exponentials derived in ref. [7]. To leading order one has

$$
e^{-J_{1} \alpha_{-} \Phi} e^{-J_{2} \alpha_{-} \Phi} \sim e^{-\left(J_{1}+J_{2}\right) \alpha_{-} \Phi}
$$

Clearly these properties are natural generalizations of the classical features recalled in section 6 .

\subsection{The quantum Hirota equation}

At present, we cannot yet write the direct quantum equivalent of Eq.6.8. Instead, we will characterize the way in which the information of the quantum Hirota equation is encoded into the theory. For this purpose, we return to Eq.6.9, which is certainly equivalent to the classical Hirota equation Eq.6.8. On the other hand, Eq.6.9 should possess meaning on the quantum level, if the role of the Taylor expansion is taken over by the operator product. The OPE of the $J=1 / 2$ Liouville exponential has the form [7]

$$
\begin{gathered}
e^{-\frac{1}{2} \alpha_{-} \Phi\left(z_{1}, \bar{z}_{1}\right)} e^{-\frac{1}{2} \alpha_{-} \Phi\left(z_{2}, \bar{z}_{2}\right)} \sim\left[\left(z_{1}-z_{2}\right)\left(\bar{z}_{1}-\bar{z}_{2}\right)\right]^{-h / 2 \pi} e^{-\alpha_{-} \Phi\left(z_{2}, \bar{z}_{2}\right)} \\
+\left[\left(z_{1}-z_{2}\right)\left(\bar{z}_{1}-\bar{z}_{2}\right)\right]^{1+3 h / 2 \pi} c_{0}+\text { descendants }
\end{gathered}
$$

where $c_{0}$ is a constant that can be changed by a global shift of the Liouville field. The second Liouville exponential is equal to a constant, since its spin $J$ is equal to zero. This property is the quantum equivalent of Eq.6.9. Thus, the quantum Hirota equation should be equivalent to the fact that for $J=0, \exp \left(-J \alpha_{-} \Phi\right)=$ cst. One sees that choosing a particular combination of derivatives in a bilinear classical expression of $J=1 / 2$ Liouville exponentials is replaced by picking up the spin zero term in the operator-product expansion of $\exp \left(-(1 / 2) \alpha_{-} \Phi\right)$ with itself. Note that

\footnotetext{
${ }^{16}$ Related formulae are already given in ref.[35], p. 27.
} 
due to the quantum effects, the difference of the powers of $\left(z_{1}-z_{2}\right)\left(\bar{z}_{1}-\bar{z}_{2}\right)$ between the first and second term is not equal to one - it is equal to $1+2 h$ - so that a simple antisymmetrization is not enough, as in the classical case, to remove the first term. Clearly, Eq.7.31 gives non-trivial equations relating the matrix elements of the quantum Liouville exponentials.

\section{Conclusions/Outlook}

The operator approach to Liouville Theory, which originated more than ten years ago, has come a long way. Starting from the analysis of the simplest Liouville field - the inverse square root of the metric - which corresponds to the $J=1 / 2$ representation, it has now progressed to the construction of the most general Liouville operators in the standard (weak coupling) regime, corresponding to arbitrary highest/lowest weight representations of the quantum group. The underlying chiral algebra, either in its Bloch wave/Coulomb gas or its quantum group covariant guise, has revealed beautiful structures which may find applications also in very different contexts. Though the completion of the quantization program of Gervais and Neveu thus finally comes into sight for the weak coupling sector, there remains an important complex of questions yet to be addressed. While the Coulomb gas picture presented here leads immediately to integral representations of arbitrary npoint functions in the half-integer positive spin case [21], the correlators of operators with continous spins require more care. This is due to the fact that outside the halfinteger positive $J$ region, the sums representing the Liouville exponentials become infinite, and their evaluation within correlation functions is quite nontrivial even in the simplest case of the three-point function [31]. In ref. [7], where the computation of three-point functions relevant for minimal matter coupled to gravity was discussed (here $J$ is half-integer negative), these difficulties were avoided in an interesting way by using instead of the "canonical" expression for the Liouville exponential as given by Eq.5.14 another operator with the same conformal weight, which is however represented by a finite sum. This approach appears to be closer in spirit to the analytic continuation procedure employed in the path integral framework, and its connection with the first-principle approach along the lines of this review certainly deserves a better understanding.

The techniques used in this work are applicable not only to the standard weak coupling $(C>25)$ regime considered here, but also to the strong coupling theory as developed in refs. [6] [17]. In this case, however, it turns out that one needs inverse powers of the screening charges Eq.3.3. These can be immediately formulated in the Gervais-Neveu framework, as the replacement $A \rightarrow-1 / A$ which inverts the screening charge just corresponds to exchanging the free field $\vartheta_{1}$ with $\vartheta_{2}$. Using the polynomial equations, and the fact that $S(\sigma) S^{-1}(\sigma)=1$ (without renormalization) it is actually possible to relate the braiding of negative powers of the screenings to that of positive powers, and one can rigorously control the exchange algebra resp. locality properties of the strong coupling operators. (This will be explained elsewhere in detail). 
In the last section, we have been discussing a possible approach to the problem of defining a $\tau$ function for the quantum Liouville theory, thus embedding our treatment into the general framework of integrable systems. We have seen that the classical Leznov-Saveliev formula has an immediate and very natural quantum generalization. The emerging quantum $\tau$ function, due to its operator nature, is a noncommutative object and this is compatible with the interesting results of [39] which however on the quantum level are at present still restricted to a subset of solutions of the full dynamics. On the other hand, our analysis is also still somewhat incomplete, as we have not yet derived the quantum Hirota equation in a satisfactory form. Given the fact that our classical Hirota equation is directly equivalent to the Liouville equation, we expect that the quantum Hirota equation must be related in a similar way to the (known) quantum Liouville equation. From that point of view, one would actually not expect that the differential equation becomes replaced by a q-difference equation on the quantum level, as in [39]. Certainly, these questions deserve further investigation.

\section{Acknowledgements}

We are grateful to E. Cremmer, D. Lebedev, J.-F. Roussel and G. Weigt for useful discussions.

\section{A Some previous results.}

This section is meant as a brief account of some previous reults, for the reader who has already some familiarity with the present approach. For lack of space, we refer the reader to refs. [2], [8] for details on the conventions used. In refs. [1, 2] the quantum group structure was shown to be of the type $U_{q}(s l(2)) \odot U_{\widehat{q}}(s l(2))$ for half-integer positive spins, where $h$ is given by Eq.2.19, and

$$
\widehat{h}=\frac{\pi}{12}(C-13+\sqrt{(C-25)(C-1)}),
$$

Each quantum group parameter is associated with a screening charge by the relations $h=\pi\left(\alpha_{-}\right)^{2} / 2, \widehat{h}=\pi\left(\alpha_{+}\right)^{2} / 2$. The basic family of $(r, s)$ chiral operators in $2 \mathrm{D}$ gravity may be labelled by two quantum group spins $J$ and $\widehat{J}$, with $r=2 \widehat{J}+1, s=2 J+1$, so that the spectrum of Virasoro weights is given by

$$
\Delta_{J \widehat{J}}=\frac{C-1}{24}-\frac{1}{24}((J+\widehat{J}+1) \sqrt{C-1}-(J-\widehat{J}) \sqrt{C-25})^{2},
$$

in agreement with Kac's formula. One outcome of ref.[B] was the fusion and braiding of the general chiral operators $V_{\underline{m}}^{(\underline{J})}$, also denoted $V_{m \widehat{m}}^{(J \widehat{J})}$, where underlined symbols denote double indices $\underline{J} \equiv(J, \widehat{J}), \underline{m} \equiv(m, \widehat{m})$, which were all taken to be half integers:

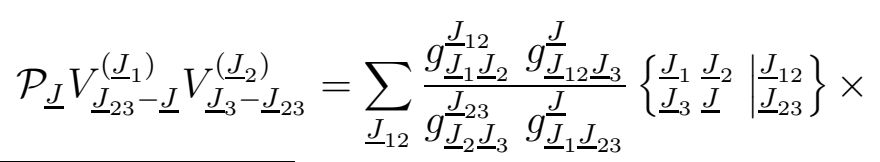

\footnotetext{
${ }^{17}$ there, and in ref. [41], also the concept of a commuting $\tau$ function is discussed
} 


$$
\begin{aligned}
& \mathcal{P}_{\underline{J}} \sum_{\{\nu\}} V_{\underline{J}_{3}-\underline{J}}^{\left(\underline{J}_{12},\{\nu\}\right)}<\varpi_{\underline{I}_{12}},\{\nu\}\left|V_{\underline{J}_{2}-\underline{J}_{12}}^{\left(\underline{J}_{1}\right)}\right| \varpi_{\underline{J}_{2}}>. \\
& \mathcal{P}_{\underline{J}} V_{\underline{J}_{23}-\underline{J}}^{\left(\underline{J}_{1}\right)} V_{\underline{J}_{3}-\underline{J}_{23}}^{\left(\underline{J}_{2}\right)}=\sum_{\underline{J}_{13}} e^{ \pm i \pi\left(\Delta_{\underline{J}}+\Delta_{\underline{J}_{3}}-\Delta_{\underline{J}_{23}}-\Delta_{\underline{J}_{13}}\right)} \times
\end{aligned}
$$

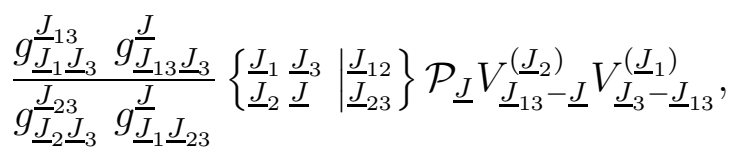

In these formulae, world-sheet variables are omitted, and $\varpi$ is the rescaled zeromode momentum of $\vartheta_{1}$ as in Eq.2.18. It characterizes the Verma modules $\mathcal{H}(\varpi)$, spanned by states noted $\mid \varpi,\{\nu\}>$, where $\{\nu\}$ is a multi-index. In the generic case, where the Verma module is trivial, $\mathcal{H}(\varpi)$ is a Fock space generated by the non-zero modes of the free field $\vartheta_{1}$ (or equivalently of $\vartheta_{2}$ ), with the ground state $\mid \varpi>$. The symbol $\varpi_{J}$ stands for $\varpi_{0}+2 J+2 \widehat{J} \pi / h$ where $\varpi_{0}=1+\pi / h$, and $\mathcal{P}_{\underline{J}}$ is the projector on $\mathcal{H}\left(\varpi_{\underline{J}}\right)$. The above formulae contain the recoupling coefficients for the quantum group structure $U_{q}(s l(2)) \odot U_{\widehat{q}}(s l(2))$, which are defined by

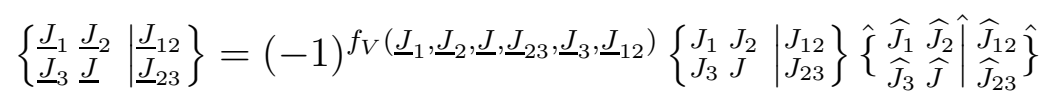

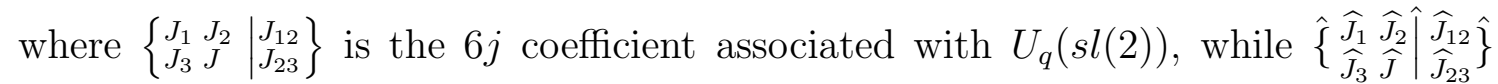
stands for the $6 j$ associated with $U_{\widehat{q}}(s l(2))$. $f_{V}\left(\underline{J}_{1}, \underline{J}_{2}, \underline{J}_{2}, \underline{J}_{23}, \underline{J}_{3}, \underline{J}_{12}\right)$ is an integer given by

$f_{V}\left(\underline{J}_{1}, \underline{J}_{2}, \underline{J}_{123}, \underline{J}_{23}, \underline{J}_{3}, \underline{J}_{12}\right)=2 \widehat{J}_{2}\left(J_{12}+J_{23}-J_{2}-J_{123}\right)+2 J_{2}\left(\widehat{J}_{12}+\widehat{J}_{23}-\widehat{J}_{2}-\widehat{J}_{123}\right)$.

In addition to these group theoretic features there appear the coupling constants $g_{\underline{J}_{1} \underline{J}_{2}}^{\underline{J_{1}}}$ whose expression was given in ref. [8]. In order to connect with the general setting recalled in section 2, let us indicate that $V_{-J, 0}^{(J, 0)}$ is proportional to $\left.f_{-J}^{J}\right|_{\text {qu }}$, and that $V_{J, 0}^{(J, 0)}$ corresponds to the normal ordered exponential of $\vartheta_{2}$, that is to $\left.f_{J}^{J}\right|_{\mathrm{qu}}$. One may verify - this is left as an exercise to the dedicated reader - that the equations just written are such that the braiding of these fields is simply

$$
\begin{aligned}
& V_{-J_{1}, 0}^{\left(J_{1}, 0\right)}\left(\sigma_{1}\right) V_{-J_{2}, 0}^{\left(J_{2}, 0\right)}\left(\sigma_{2}\right)=e^{-2 i h J_{1} J_{2} \epsilon\left(\sigma_{1}-\sigma_{2}\right)} V_{-J_{2}, 0}^{\left(J_{2}, 0\right)}\left(\sigma_{2}\right) V_{-J_{1}, 0}^{\left(J_{1}, 0\right)}\left(\sigma_{1}\right), \\
& V_{J_{1}, 0}^{\left(J_{1}, 0\right)}\left(\sigma_{1}\right) V_{J_{2}, 0}^{\left(J_{2}, 0\right)}\left(\sigma_{2}\right)=e^{-2 i h J_{1} J_{2} \epsilon\left(\sigma_{1}-\sigma_{2}\right)} V_{J_{2}, 0}^{\left(J_{2}, 0\right)}\left(\sigma_{2}\right) V_{J_{1}, 0}^{\left(J_{1}, 0\right)}\left(\sigma_{1}\right) .
\end{aligned}
$$

where $\epsilon\left(\sigma_{1}-\sigma_{2}\right)$ is the sign of $\sigma_{1}-\sigma_{2}$ (for definiteness, we consider the interval $\left.0 \leq \sigma_{i} \leq 2 \pi\right)$. This confirms that they are normal ordered exponentials of free fields, in agreement with the starting point of the GN quantization. Note that the braiding of $V_{-J, 0}^{(J, 0)}$ with $V_{J, 0}^{(J, 0)}$ involves the full complexity of the 6-j coefficients, so that the commutation relations of $\vartheta_{1}$ with $\vartheta_{2}$ are definitely not of free-field type.

\section{References}

[1] O. Babelon, Phys. Lett. B215 (1988) 523 . 
[2] J.-L. Gervais, Comm. Math. Phys. 130 (1990) 257.

[3] J.-L. Gervais, Phys. Lett. B243 (1990) 85 .

[4] E. Cremmer, J.-L. Gervais, Comm. Math. Phys. 144 (1992) 279 .

[5] J.-L. Gervais, Int. J. Mod. Phys. 6 (1991) 2805.

[6] J.-L. Gervais, Comm. Math. Phys. 138 (1991) 301.

[7] J.-L. Gervais, "Quantum group derivation of 2D gravity-matter coupling" Invited talk at the Stony Brook meeting String and Symmetry 1991, Nucl. Phys. B391 (1993) 287.

[8] E. Cremmer, J.-L. Gervais, J.-F. Roussel, Nucl. Phys. B413 (1994) 244.

[9] E. Cremmer, J.-L. Gervais, J.-F. Roussel, Comm. Math. Phys. 161 (1994) 597.

[10] O. Babelon, Comm. Math. Phys. 139 (1991) 619.

[11] J.-L. Gervais, A. Neveu, Nucl. Phys. B238 (1984) 125; ibid., p. 396.

[12] J.-L. Gervais, J. Schnittger, Phys. Lett. B315 (1993) 258

[13] A.A. Belavin, A.M. Polyakov, A.B. Zamolodchikov, Nucl. Phys. B241 (1984) 332.

[14] Vl. Dotsenko, V. Fateev, Nucl. Phys. B251 (691) (1985) .

[15] G. Moore, N. Seiberg, Comm. Math. Phys. 123 (1989) 77.

[16] J.-L. Gervais, Y. Matsuo, Phys. Lett. B274 (1992) 309; Comm. Math. Phys. 152 (1993) 317.

[17] J.-L. Gervais, J.-F. Roussel, Nucl. Phys. B426 (1994) 140.

[18] A. Anderson, B.E.W. Nilsson, C.N. Pope and K.S.Stelle, Nucl. Phys. B430 (1994) 107.

[19] J.-L. Gervais, A. Neveu, Nucl. Phys. B224 (1983) 329.

[20] J.-L. Gervais, A. Neveu, Nucl. Phys. B209 (1982) 125.

[21] D. Lüst, J. Schnittger, Int. J. Mod. Phys. A6 (1991) 3625; J. Schnittger, Ph.D. thesis, Munich 1990.

[22] L. Johannson, A. Kihlberg, R. Marnelius, Phys. Rev. D 29 (1984) 2798; L. Johannson, R. Marnelius, Nucl. Phys. B254 (1985) 201.

[23] see, e. g. M. Jimbo, T. Miwa, Publications of the R.I.M.S. vol 19, No 3, 1983. 
[24] V. Kač, M. Wakimoto, Proc. Symp. Pure Math. 49 (1989) 191.

[25] J.-L. Gervais, J. Schnittger, Nucl. Phys. B413 (1994) 277.

[26] J.-L. Gervais, J. Schnittger, Nucl. Phys. B431 (1994) 273.

[27] H.J. Otto, G. Weigt, Phys. Lett. B159 (1985) 341; Z. Phys. C31, (1986) 219; G. Weigt, "Critical exponents of conformal fields coupled to two-dimensional quantum gravity in the conformal gauge", talk given at 1989 Karpacz Winter School of Theor. Physics, preprint PHE-90-15; G. Weigt, "Canonical quantization of the Liouville theory, quantum group structures, and correlation functions", talk given at 1992 Johns Hopkins Workshop on Current Problems in Particle Theory, Goteborg, Sweden, hep-th 9208075, print-92-0383 (DESY-IFH).

[28] E.Braaten, T.Curtright, C.Thorn, Phys. Lett. B118 (1982) 115; Phys. Rev. Lett. 48 (1982) 1309; Ann. Phys. (N.Y.) 147 (1983) 365; E.Braaten, T.Curtright, G.Ghandour, C.Thorn, Phys. Rev. Lett. 51 (1983) 19; Ann. Phys. (N.Y.) 153 (1984) 147.

[29] G. Jorjadze, A. Pogrebkov, M. Polivanov, Teor. Mat. Fiz. 40 (1979) 221; A. Pogrebkov, Teor. Mat. Fiz. 45 (1980) 161; G. Jorjadze, A. Pogrebkov, M. Polivanov, S. Talalov, J. Phys. A: Math. Gen. 19 (1986) 121.

[30] J. Balog and L. Palla, Phys. Lett. B274 (1992) 232.

[31] J. Schnittger, proceedings of the 1993 NATO adv. research workshop on new developments in string theory, conformal models and field theory, Cargese May 93, Plenum Press, to appear.

[32] Vl. Dotsenko, V. Fateev, Nucl. Phys. B251 (1985) 691.

[33] G. Felder, Nucl. Phys. B317 (1989) 215; erratum Nucl. Phys. B324 (1989) 548 .

[34] A. N. Leznov, M. V. Saveliev, Phys. Lett. B79 (1978) 294; Lett. Math. Phys. 3 (1979) 207; Comm. Math. Phys. 74 (1980) 111; Lett. Math. Phys. 6 (1982) 505; Comm. Math. Phys. 89 (1983) 59; Group-Theoretical Methods for Integration of Nonlinear Dynamical Systems. Progress in Physics v. 15, Birkhauser-Verlag, 1992.

[35] A. N. Leznov, M. V. Saveliev, Acta Applicandae Mathematicae 16 (1989) 1.

[36] K. Kajiwara, J. Satsuma, J. Phys. Soc. Jpn., 60 (1991) 3986; K. Kajiwara, Y. Ohta, J. Satsuma, "q-Discrete Toda Molecule Equation", solv-int/9304001.

[37] F. Smirnov, "What are we quantizing in integrable field theory", hepth/9307097. 
[38] A. Gerasimov, S. Khoroshkin and D. Lebedev, "q-deformations of $\tau$ functions: a toy model", talk presented at the P. Cartier seminar at Ecole Normale Superieure, Paris, 1993.

[39] A. Gerasimov, S. Khoroshkin, D. Lebedev, A. Mironov, A. Morozov, ITEP-M2-94, hep-th 9405011; A. Mironov, FIAN-TD-12-94, hep-th 9409190.

[40] S. Kharchev and A. Mironov, " $\tau$ function of two-dimensional Toda lattice and quantum deformations of integrable hierarchies", FIAN-TD-02-93.

[41] A. Mironov, A. Morozov, L. Vinet, FIAN-TD-22-93, hep-th 9312213. 